Portland State University

PDXScholar

Winter 2-23-2018

\title{
Recent Advances in Activity-Based Travel Demand Models for Greater Flexibility
}

Kihong Kim

Portland State University

Follow this and additional works at: https://pdxscholar.library.pdx.edu/open_access_etds

Part of the Transportation Commons, and the Urban Studies Commons Let us know how access to this document benefits you.

\section{Recommended Citation}

Kim, Kihong, "Recent Advances in Activity-Based Travel Demand Models for Greater Flexibility" (2018). Dissertations and Theses. Paper 4225.

https://doi.org/10.15760/etd.6109

This Dissertation is brought to you for free and open access. It has been accepted for inclusion in Dissertations and Theses by an authorized administrator of PDXScholar. Please contact us if we can make this document more accessible: pdxscholar@pdx.edu. 
Recent Advances in Activity-Based Travel Demand Models for

\title{
Greater Flexibility
}

\section{by}

Kihong Kim

A dissertation submitted in partial fulfillment of the requirements for the degree of

\author{
Doctor of Philosophy \\ in \\ Urban Studies
}

\author{
Dissertation Committee: \\ James Strathman, Chair \\ Liming Wang \\ Kelly Clifton \\ Wayne Wakeland
}

Portland State University

2018 
(C) 2018 Kihong Kim 


\begin{abstract}
Most existing activity-based travel demand models are implemented in a tour-based microsimulation framework. Due to the significant computational and data storage benefits, the demand microsimulation allows a greater amount of flexibility in terms of demographic market segmentation, temporal scale, and spatial resolution, and thus the models can represent a wider range of travel behavior aspects associated with various policies and scenarios. This dissertation proposes three innovative methodologies, one for each of the three key dimensions, to fulfill the greater level of details toward a more mature state of activity-based travel demand models.
\end{abstract}


$\begin{array}{ll}\text { Abstract } & \text { i }\end{array}$

List of Tables $\quad$ iv

List of Figures $\quad$ V

CHAPTER 1.

INTRODUCTION 1

CHAPTER 2.

DISCREPANCY ANALYSIS OF ACTIVITY SEQUENCE 9

2.1 Sequence Alignment Methods for Activity Pattern Analysis 12

2.2 Data and Methods 16

2.2.1 Sequence Representation 18

$\begin{array}{ll}\text { 2.2.2 Sequence Alignment } & 19\end{array}$

2.2.3 Discrepancy Analysis of Activity Sequences 20

2.2.4 Tree-Structured Analysis of Sequences 24

2.3 Results and Discussions 25

2.3.1 Sequence Discrepancy Analysis with Multiple Factors 25

2.3.2 Tree Analysis of Activity Sequences 26

2.4 Conclusion 31

CHAPTER 3.

DISCRETE-TIME ACTIVITY DURATION ANALYSIS 33

$\begin{array}{lll}3.1 & \text { Background } & 38\end{array}$

3.1.1 A Discrete-Time Duration Model for a Single Event 38

3.1.2 A Discrete-Time Duration Model for Recurrent Events 41

3.1.3 Modeling Competing Risks and Multiple States 42

3.2 Application to Activity Duration Analysis 44

3.2.1 Sample Data Definition $\quad 44$

3.2.2 Data Transformation 46 
3.3 Results

3.3.1 Random Effects

3.3.2 Fixed Effects

3.4 Conclusion and Future Work

CHAPTER 4.

MULTIPLE IMPUTATION BY CHAINED EQUATIONS 60

4.1 Background $\quad 62$

4.1.1 Patterns and Mechanisms of Missing Data 62

4.1.2 Multiple Imputation 64

4.1.3 Multiple Imputation by Chained Equations (MICE) 66

4.1.4 Recursive Partitioning in MICE 69

4.2 Case Study: Parcel Data Imputation $\quad 70$

4.2.1 Setting Up MICE: Choice of Predictors 72

4.2.2 Setting Up MICE: Choice of Imputers 75

$\begin{array}{ll}4.2 .3 \text { Visual Diagnosis } & 75\end{array}$

$\begin{array}{lll}4.3 & \text { Assessment of MICE } & 78\end{array}$

$\begin{array}{lll}4.4 & \text { Discussion } & 81\end{array}$

CHAPTER 5.

CONCLUSION

$\begin{array}{ll}\text { REFERENCES } & 87\end{array}$ 


\section{List of Tables}

Table 2-1 Applications of Sequence Alignment Methods to Activity Pattern Analysis ...13

Table 2-2 Activity Aggregation and Average Duration (1,000 Persons) ........................17

Table 2-3 Standard ANOVA vs. Generalized MANOVA: One-Way Design..................23

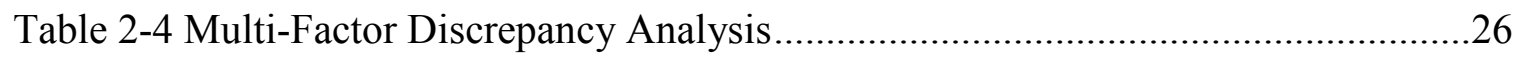

Table 3-1 Frequency and Average Duration of Activity Episodes for Retirees ...............46

Table 3-2 Transitions of 2,469 Activity Episodes from Origin to Destination ................47

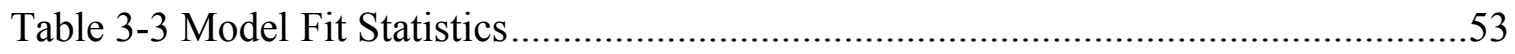

Table 3-4 Random Effects Variance-Covariance Matrix ..............................................54

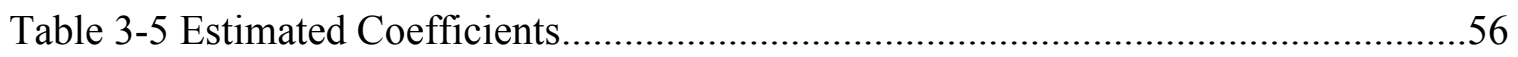

Table 4-1 Missing Data Pattern of 271,977 Parcels $(1=$ observed $\& 0=$ missing $)$.........72

Table 4-2 A List of Predictors Included in the MICE Imputers ....................................74

Table 4-3 Missing Data Pattern of 227,591 Parcels in the Training Data Set ..................79

Table 4-4 Validation Results of MICE (PMM vs. CART vs. Random Forests) ..............81 


\section{List of Figures}

Figure 2-1 Activity Sequence Index Plot and Activity State Distribution Plot .................19

Figure 2-2 Induction Trees of Activity Sequences: .......................................................30

Figure 3-1 Converting from Individual-Episode to Individual-Episode-Period.................49

Figure 4-1 Visual Diagnosis of the CART-based MICE .................................................77 


\section{INTRODUCTION}

Transportation planning agencies have long developed travel forecasting models to evaluate the impacts of future changes in economy, demography, land use, and/or transportation on the performance of a regional transportation system. The first generation of travel forecasting models typically consists of four sequential steps: trip generation, trip distribution, modal split, and trip assignment. The first three steps are grouped as travel demand models, which collectively estimate the travel demand of regional residents, the travel demand for goods movement, and/or the travel demand for special purposes. The last step, also known as network supply models, measures transportation performance, such as link volume, link speed, link travel time, etc. (Castiglione, Bradley, \& Gliebe, 2015).

Since 1980s the travel demand models have slowly evolved from trip-based to activity-based approaches as transportation planning and policies in the United States has expanded from "long-term supply-oriented mobility" to "short-term demand-oriented accessibility" (Pinjari \& Bhat, 2011). The shortcomings of the trip-based travel demand models are well recognized in both theory and practice. In the trip-based approach, the unit of analysis for modeling travel is a trip that connects two locations. Therefore, the trip-based models essentially ignore the interactions between trips made within the same trip chain, the interactions between trip chains made for the same day, and the interactions between trips made by household members (Vovsha, Bradley, \& Bowman, 2005). In addition, the trip-based approach forces a quite simple overall model structure. 
This is mainly because the trip-based models are inevitably implemented by an aggregate method called zonal enumeration or fractional probability (Bradley, Bowman, \& Lawton, 1999). In other words, all possible combinations of the outcomes of different demand sub-models are enumerated and the sub-model probabilities are multiplied to distribute demand across all the alternatives. Donnelly, Erhardt, Moeckel and Davidson (2010) illustrates how quickly the problem size increases in the aggregate implementation method as the complexity of the sub-models is added. Indeed, in the trip-based approach, adding more dimensions to travel demand sub-models is often not practical because it becomes computationally intensive and cumbersome to manage. As a result, there is a limit in alleviating aggregation biases with respect to demographic market segmentation, temporal scale, and spatial resolution.

On the other hand, the activity-based approach is typically implemented on a tourbased microsimulation framework (Davidson, et al., 2007). On the tour-based framework, the basic travel unit is not a trip any more but a tour that is defined as a chain of trips starting and ending at home. The main advantage of the tour-based structure is to preserve a consistency among multiple trips within a tour in terms of travel mode, destination, and timing. On the microsimulation framework, a full list of households and persons in a synthetic population is simulated during a simulation day. Compared with zonal enumeration used for the trip-based models, the demand microsimulation provides significant benefits of computation and data management, which allows for more sophisticated travel demand sub-model developments. As there is virtually no limit to the number of predictors for the core probability demand sub-models, the true advantage of 
activity-based travel demand models is to allow a greater amount of flexibility in terms of demographic market segmentation, temporal scale, and spatial resolution, and thus the models can represent a wider range of travel behavior aspects associated with various transportation planning and policies.

In this dissertation, three innovative methodologies, one for each of the three key dimensions, are proposed to fulfill the greater level of details toward a more mature state of activity-based travel demand models. For each of the proposed methods, this chapter provides an overview of research background and motivation and summarizes key features of the method. The subsequent three chapters will describe the methods as standalone articles. Then the dissertation will conclude with a discussion of how they contribute to the current literature.

\section{Discrepancy Analysis of Activity Sequences}

In the trip-based models, demographic market segments are pre-defined with a very limited number of population groups, and this market segmentation is usually held constant across all the demand sub-models. For the activity-based models, however, it is unnecessary to pre-define demographic segments because individual households and persons in a synthetic population are simulated (Castiglione et al., 2015). Any known attributes that characterize individual households and persons can be used in the models, including household size, age of household head, household income, number of household workers, number of household students, number of household children, person age, person sex, person employment status, and many others. 
It was an important topic in the early stage of the activity-based travel behavior analysis to identify meaningful attributes of households and persons that help explain how different households and persons make different activity-travel decisions (Pas, 1983). The holistic approach to understanding complex activity patterns has recently received new attention since the introduction of sequence alignment methods into time use and transportation research (Wilson, 1998). In existing literature, sequence alignment methods are almost always combined with cluster analysis. Although this cluster-based approach has been proven powerful for discovering a typology of activity patterns, it does not seem successful in identifying various contexts that would impact the patterns. This is because cluster analysis may cause too much information loss as a result of reducing a large set of observations into a limited number of clusters.

Instead of the cluster-based approach, a new methodological combination of sequence alignment with discrepancy analysis is proposed in this dissertation. As a generalization of the principle of ANOVA, discrepancy analysis enables to evaluate the association between complex objects (e.g., activity sequences) characterized by a pairwise distance matrix and one or more covariates. The proposed method was originally developed in ecology under the name of a non-parametric MANOVA (Anderson, 2001), and recently introduced into sociology with the name of discrepancy analysis (Studer, Ritschard, Gabadinho, \& Müller, 2011) and ANODI (Bonetti, Piccarreta, \& Salford, 2013). Additionally, an induction tree is built to visualize how individual activity sequences vary with the value of covariates. 


\section{Discrete-Time Analysis of Activity Duration}

As one of the core components in activity-based travel demand models, activity scheduling models activity starting and ending times and activity duration. There are two primary approaches for representing the process of activity scheduling: "rubber-banding" and "growing" approaches (Gliebe \& Kim, 2010). The rubber-banding scheduling predefines an overall daily tour pattern, and then fills the entire day by adding intermediate activity stops, which is also known as hierarchical scheduling. In the growing or chronological scheduling, it is assumed that as the day goes by, people decide what to do next, where to do it, and how to get there. These sequential decisions depend on previous activities, time windows, business hours, intra-household interactions, and so on.

On the other hand, the integration between activity-based demand models and dynamic network supply models recently becomes a key issue. Two approaches are available for the demand-supply integration: sequential integration and dynamic integration (Konduri, 2012). In the traditional sequential integration approach, the demand and supply components are run independently and sequentially in the form of an "input-output data flow" with a feedback of the network conditions until a convergence is achieved. The dynamic integration approach adopts an "event-based paradigm" and constantly communicates between the demand and supply models along a continuous time axis. Under the dynamic integration approach, the dynamic traffic assignment (DTA) is better integrated with the growing scheduling of activity participations than the rubber-banding scheduling (Gliebe \& Kim, 2010). For the growing scheduling, activity 
duration can be explicitly incorporated at a continuous or quasi-continuous temporal scale by hazard-based duration models that deal with time to an event.

In this dissertation, a discrete-time hazard-based duration model is proposed, which is essentially equivalent to a discrete choice model with temporal dummies as the simplest form of dynamic discrete choice models. There are several important benefits in the discrete-time duration models over the continuous-time models. It is possible in the discrete-time framework to handle tied observations (e.g., joint activity participations of household members) and to add time-varying covariates (e.g., transit operating hours and business hours). In addition, the discrete-time method relatively easily expands the model structure for more complex situations. In the application of the discrete-time duration model to activity duration analysis, complex situations of activity-travel data need to be considered, including multiple states of origin activity, competing risks of destination activity, and a multilevel structure for recurrent activity episodes within individuals. Moreover, a circular or periodic variable is introduced as a combination of sine and cosine in order to model time-of-day effects.

\section{Multiple Imputation by Chained Equations}

In recent activity-based model developments it becomes more common to use multiple spatial scales within a model system (Castiglione et al., 2015). For example, larger spatial units, such as traffic analysis zones (TAZs) that are often defined at a resolution that is similar to Census tracts or Census block groups, can be used to develop auto and transit network skims. At the same time, one can use smaller spatial units, such as Census blocks 
or parcels, to incorporate the attributes of small-scale land use and transportation systems (e.g., the mix of land uses and the distance to transit stops).

However, it is more difficult to develop, maintain and forecast the attributes of smaller spatial units than those of TAZs. Especially, parcels data are often incomplete with missing values. According to the study of Waddell, Peak and Caballero (2004), up to $70 \%$ of the efforts in land use and transportation modeling are spent on data processing, and handling missing data is a major piece of these efforts. In the field of integrated land use and transportation modeling, missing values are largely handled in an ad-hoc basis without assessment of imputation quality. Recently, more sophisticated data imputation techniques based on machine learning have been developed in other fields such as statistics and computer science.

This dissertation introduces Multiple Imputation by Chained Equations (MICE) and tests it with parcel data. Instead of generating a single best guess, this technique replaces each missing value with a set of plausible values. The MICE is flexible and practical because it can handle a mix of continuous and discrete missing variables by imputing each of the missing variables based on its own imputation engine. In practice, the success of a MICE application depends on how to design well for the imputation engine. Recently, the use of recursive partitioning as the imputation engine of MICE becomes popular because it can capture complex interaction effects on missing variables with minimal effort to set up the models. As a case study, the MICE approach is used for parcel data imputation. The performance of MICE is evaluated with two recursive 
partitioning methods (i.e., Classification and Regression Trees and Random Forests) using a cross-validation technique. 


\section{DISCREPANCY ANALYSIS OF ACTIVITY SEQUENCE}

Over the past four decades the goals of urban transportation planning and policies have shifted from meeting 'long-term, supply-oriented, mobility' needs to facilitating 'shortterm, demand-oriented, accessibility' needs. This shift has created a new paradigm, called activity-based travel behavior analysis (Pinjari \& Bhat, 2011). The underlying theory of the activity-based analysis recognizes that travel is a derived demand to participate in activities that are separated in time and space. The theory implies that "travel can be best understood in the broader context of activity patterns" (Ettema, 1996). An individual's activity pattern is a complex phenomenon resulting from interactions of multiple dimensions, such as timing, duration, locations, activity types, travel mode, trip chaining, activity jointness, activity substitution, activity priority, activity planning horizon, and so on (Burnett \& Hanson, 1982).

Given that it is very difficult to capture their full complexities with all the dimensions, two general approaches have been used to measure the complexity of activity-travel patterns (Burnett \& Hanson, 1982). One is decomposing an individuals' activity pattern into the numerous dimensions and generating separate measures for each of the dimensions. The other is treating the pattern as a multidimensional 'holistic' entity. Currently, the first approach is dominant in activity pattern research, in part because most existing operational activity-based travel forecasting systems are implemented on a micro-simulation framework that consists of a series of calibrated econometric models to address the multiple dimensions either individually or jointly (Davidson, et al., 2007). 
Discrete choice models, discrete-continuous choice models, and hazard-based duration models are widely used as the basis of the micro-simulation implementation framework (Pas, 1997). The second approach to measuring activity patterns, which relates to this paper, was popular in the early stage of the activity-based travel behavior analysis. Many transportation researchers recognized early on the complexity of activity patterns and emphasized the need to understand individual activity patterns as a whole (Pas, 1983; Koppelman \& Pas, 1985; Recker, McNally, \& Root, 1985). The holistic approach focuses more on interpreting people's daily or weekly activity patterns into homogeneous groups and identifying determinants or constraints that influence the homogeneous patterns.

The early efforts on the holistic approach fall into two categories (Pas, 1983). First, each activity pattern is described by numerous measures, and then the measures are used for factor analysis or principal components analysis to identify its salient features. The latter information is often used to classify the whole set of activity patterns into a small number of similar groups. The second holistic method is comparing individuals' activity-travel patterns each other that are often represented on time-slice variables. The comparison produces a matrix of pairwise dissimilarities between the patterns, which is subsequently used for cluster analysis.

Given our limited knowledge about human activity decisions, both atomistic and holistic approaches to accounting for the complexity of activity patterns are equally important and complementary (Burnett \& Hanson, 1982). The atomistic or decomposing approach serves as a core part of activity-based models to predict the patterns, while the holistic approach provides theoretical and empirical foundations by identifying travel 
determinants. However, it is surprising that although attention to the former is prominent today, the latter has seen little progress since the development of the cosine similarity index by Koppelman and Pas (1985) and the feature extraction of Recker, McNally and Root (1985), even if those classifications may be not satisfactory in that they only explain a small amount of variability within the clusters (Schlich \& Axhausen, 2003).

The holistic approach to understanding complex activity patterns has recently received new attention since the introduction of sequence alignment methods into time use and transportation research (Wilson, 1998). In existing literature, sequence alignment methods are almost always combined with cluster analysis. Although this cluster-based approach has been proven powerful for discovering a typology of activity patterns, it does not seem successful in identifying various contexts that would impact the patterns. This is because cluster analysis may cause too much information loss as a result of reducing a large set of observations into a limited number of clusters (Studer, et al., 2011). Consequently, we need a direct analysis of the association between pairwise dissimilarity measures and explanatory variables without any prior clustering. Inspired from the work of Studer et al. (2011), this paper proposes a new combination of sequence alignment with ANOVA-like tools, not with cluster analysis. The proposed method was originally developed in ecology under the name of a non-parametric MANOVA (Anderson, 2001), and recently introduced into sociology with the name of discrepancy analysis (Studer, et al., 2011) and ANODI (Bonetti, et al., 2013). In addition to the methodological combination, an induction tree is built to visualize how activity sequences may vary with the value of covariates. 


\subsection{Sequence Alignment Methods for Activity Pattern Analysis}

Sequence alignment methods, also known as optimal matching (OM), measure the dissimilarity between two sequences of characters by calculating the minimal cost of transforming one sequence into the other (Wilson, 1998; Martin \& Wiggins, 2011). Two basic operations are used in the sequence transformation: substitutions and indels. The substitution operation replaces an element of one sequence with the different element located at the same position of the other sequence. The indel, an OM jargon standing for the insertion or deletion of an element, causes a one-position movement of all the elements to the right. The transformation costs for substitutions and indels are assigned by the researcher in advance either theoretically or empirically. A dynamic programming algorithm is usually used to repeat the sequence alignment for all pairs of sequences. The final output of sequence alignment is a pairwise distance matrix of activity sequences, which is almost always used as input for cluster analysis. The resulting cluster membership is often associated with other variables, either as a dependent variable (e.g., multinomial logit models) or as an independent variable (e.g. ANOVA).

Sequence alignment methods were initially developed in biology in 1970s from the needs of analyzing DNA sequences of nucleic acids or protein sequences of amino acids, and introduced into social science in the 1980s (Abbott \& Tsay, 2000). It was in the late 1990s that the methods were first adopted for the analysis of people's activity patterns (Wilson, 1998). Recently, sequence alignment is applied in the goodness-of-fit testing for activity-based travel demand micro-simulation models, in which predicted activity-travel patterns are compared with the observed one at an agent level (Sammour, 
et al., 2012). Table 1 summarizes some applications of sequence alignment methods to activity pattern analysis.

Table 2-1 Applications of Sequence Alignment Methods to Activity Pattern Analysis

\begin{tabular}{|c|c|c|c|c|c|c|}
\hline \multirow{2}{*}{ Citation } & \multicolumn{2}{|c|}{$\begin{array}{l}\text { Sequence } \\
\text { Specification }\end{array}$} & \multicolumn{2}{|c|}{$\begin{array}{c}\text { Assigning } \\
\text { Transformation Costs }\end{array}$} & \multirow{2}{*}{$\begin{array}{c}\text { Number } \\
\text { of } \\
\text { Clusters }\end{array}$} & \multirow{2}{*}{$\begin{array}{c}\text { Subsequent } \\
\text { Data Analysis }\end{array}$} \\
\hline & $\begin{array}{l}\text { sequence } \\
\text { element }\end{array}$ & $\begin{array}{l}\text { time } \\
\text { scale }\end{array}$ & substitutions & indels & & \\
\hline \multirow{2}{*}{$\begin{array}{l}\text { (Wilson, } \\
\text { 2001) }\end{array}$} & activity only & $30 \mathrm{~min}$ & $\begin{array}{l}\text { varying by } \\
\text { activity being } \\
\text { compared }\end{array}$ & $\begin{array}{l}\text { gap opening; } \\
\text { extension }\end{array}$ & 4 & ANOVA \\
\hline & $\begin{array}{c}\text { activity; } \\
\text { location; } \\
\text { person present }\end{array}$ & $30 \mathrm{~min}$ & complicated & $\begin{array}{l}\text { gap opening; } \\
\text { extension }\end{array}$ & 4 & ANOVA \\
\hline $\begin{array}{l}\text { (Shoval \& } \\
\text { Isaacson, } \\
\text { 2007) }\end{array}$ & location only & $1 \mathrm{sec}$ & not used & $\begin{array}{l}\text { gap opening; } \\
\text { extension }\end{array}$ & 3 & $\begin{array}{l}\text { contingency } \\
\text { table analysis }\end{array}$ \\
\hline $\begin{array}{c}\text { (Saneinejad } \\
\& \text { Roorda, } \\
\text { 2009) }\end{array}$ & $\begin{array}{l}\text { activity; } \\
\text { location }\end{array}$ & $15 \mathrm{~min}$ & not used & $\begin{array}{l}\text { gap opening; } \\
\text { extension }\end{array}$ & 9 & $\begin{array}{l}\text { descriptive } \\
\text { statistics }\end{array}$ \\
\hline
\end{tabular}

Wilson (2001) examined the activity patterns of 248 Canadian women who were selected as a $5 \%$ random sample from the main time-use survey. The 248 activity diaries were converted into two sets of sequences: one was composed of only one dimension (i.e., activity type) and the other had three dimensions (i.e., activity type, location and the presence of other persons). The author defined 15 activity types regardless of in-home or out-of-home, 5 locations (i.e., home, workplace, other place, traveling, or unknown), and 5 types of persons present with the survey respondent (i.e., alone, household members, friends, other persons, or unknown). Both the activity-only sequences and the activitysetting sequences were specified in 30 -min time intervals. In the activity-only sequence alignments, the substitution costs varied with the type of activities being compared. The 
indel costs were also further refined into two types; a gap open penalty for the first indel position and a gap extension penalty for all the subsequent indels. For the activitysettings sequences, the transformation costs of substitutions were more complicated due to many ways of combining levels of the activity settings, and the same indel costs were assigned as in the activity-only sequences. Both sets of sequences were visually clustered into four similar patterns. Subsequently, the author conducted ANOVA to examine discriminatory power of the cluster membership with regard to socioeconomic characteristics.

Shoval and Isaacson (2007) investigated the moving paths of visitors to the Old City of Akko in Israel. For this study, 40 visitors were given GPS devices to track their movements in space and time. The study area was divided into 26 polygons with each polygon representing a single location, and each visitor's polygon locations were consecutively recorded every second during the visit. Since visitors started their trip at different times of day and their visit durations were different each other, the sequence lengths of visitors varied. Only indel operations were used to align the location sequences of different lengths. The authors identified three distinct moving paths. In addition, they conducted a contingency table analysis.

Saneinejad and Roorda (2009) measured similarities between weekly activity sequences of 282 individuals who participated in a special survey in which, among other information, respondents were directly asked to describe activities that they normally do every week. The authors defined 10 activity types (i.e., 9 routine and 1 non-routine activities) and 2 activity locations (i.e., in home and out of home). Two letters 
representing activity type and location were specified on every 15-min time interval of 5 weekdays for each individual, and thus the length of all sequences is equal to 480 . No substitutions were used, while two types of indels were defined: gap opening indels and gap extending indels. The authors identified 9 different schedules of routine weekly activities. Then, they described socioeconomic characteristics of individuals within each cluster.

It has been demonstrated that sequence alignment methods outperform in classifying activity-travel patterns compared to other conventional dissimilarity measures, such as Euclidean distance measures and signal-processing theoretical measures (Joh, Arentze, \& Timmermans, 2001). Only sequence alignment methods can capture sequential information imbedded in activity patterns. This unique ability of sequence alignment methods may yield better cluster solutions that are more likely to be sensitive to activity-travel constraints. On the other hand, there are many controversial issues for using sequence alignment in social science, rather than in biology (Aisenbrey $\&$ Fasang, 2010). The issues include the meaning of indels and substitutions in the context of human behavior analysis; the arbitrary assignment of transformation costs for substitutions and indels; required symmetry of the pairwise distance matrix; the lack of proper support of multi-dimensional analysis; and time distortion by indels. Fortunately, a 'second wave' of sequence alignment toward methodological improvements is currently observed in sociology (Aisenbrey \& Fasang, 2010) as well as transportation (Joh, Arentze, Hofman, \& Timmermans, 2002; Wilson, 2008). 


\subsection{Data and Methods}

The Portland metropolitan portion of the 2011 Oregon Travel and Activity Survey (OTAS) is used for this study. The portion of the Survey records all locations visited by approximately 15,000 persons of nearly 6,500 households living in the Portland metropolitan region during a scheduled day. A random sample of 1,000 persons, which accounts for about $6.5 \%$ of the main survey, is selected to reduce the computational burden of performing a series of proposed methods. In addition, for simplicity reasons, 24 types of self-reported original activities are aggregated into 12 major activity types as shown in Table 2. It should be noted that all at-home activities are grouped into three different categories: home in the beginning of the day (HB), home returning temporarily in the middle of the day (HR), and home in the end of the day (HE). This categorization of at-home activities may help partially avoid time distortion that often occurs by indels. Time distortion by indels is a unique feature of sequence alignment in matching sequences of varying lengths. In case of aligning sequences involving timing and duration of episodes, the indel operations need to be carefully used to prevent excessive time distortion (Shoval \& Isaacson, 2007; Lesnard, 2010). A simpler way of avoiding time distortion, as in this study, might be roughly disaggregating a sequence state (i.e., athome activity) by time periods (i.e., in the beginning, in the middle and in the end of the day). 
Table 2-2 Activity Aggregation and Average Duration (1,000 Persons)

\begin{tabular}{|c|c|c|c|c|}
\hline $\begin{array}{l}12 \text { Activity Types } \\
\text { (Aggregated) }\end{array}$ & $\begin{array}{c}24 \text { Activity Types } \\
\text { (Originally Self-Reported) }\end{array}$ & Code & $\begin{array}{l}\text { No. of } \\
\text { Episodes }\end{array}$ & $\begin{array}{c}\text { Average } \\
\text { Duration } \\
\text { (min.) }\end{array}$ \\
\hline Home in the beginning & $\begin{array}{c}\text { work at home; } \\
\text { all other activities at-home }\end{array}$ & $\mathrm{HB}$ & 968 & 495 \\
\hline $\begin{array}{l}\text { Home returning } \\
\text { temporarily }\end{array}$ & $\begin{array}{c}\text { work at home; } \\
\text { all other activities at-home }\end{array}$ & HR & 410 & 121 \\
\hline Home in the end & $\begin{array}{c}\text { work at home; } \\
\text { all other activities at-home }\end{array}$ & $\mathrm{HE}$ & 848 & 560 \\
\hline Work & $\begin{array}{l}\text { work; all other activities at work; } \\
\text { work related }\end{array}$ & WK & 625 & 312 \\
\hline School & $\begin{array}{l}\text { attending class; } \\
\text { all other activities at school }\end{array}$ & $\mathrm{SC}$ & 218 & 355 \\
\hline Escort & $\begin{array}{l}\text { drop off; } \\
\text { pick up }\end{array}$ & $\mathrm{EC}$ & 270 & 10 \\
\hline Eat out & eat meal outside of home & EO & 196 & 44 \\
\hline Household maintenance & $\begin{array}{l}\text { routine shopping; major shopping; } \\
\text { household errands }\end{array}$ & $\mathrm{HM}$ & 516 & 28 \\
\hline Personal business & $\begin{array}{l}\text { service private vehicle; personal business; } \\
\text { health care }\end{array}$ & PB & 226 & 71 \\
\hline Social recreation & $\begin{array}{l}\text { civic/religious activities; outdoor/indoor } \\
\text { recreation; visit friends; loop trip }\end{array}$ & SR & 375 & 134 \\
\hline Other & other & OT & 4 & 387 \\
\hline Trip for the activity & $\begin{array}{l}\text { not categorized in the survey, } \\
\text { but explicitly included for this study }\end{array}$ & TR & 3656 & 19 \\
\hline
\end{tabular}

The goal of this paper is to identify determinants that influence individuals' daily activity-travel patterns from the holistic perspective. To achieve this goal, four sequential steps are proposed: 1) representing an individual's activity diary as a sequence of characters; 2) performing sequence alignment to produce a pairwise distance matrix among all activity sequences; 3 ) conducting discrepancy analysis to examine the association between activity sequences characterized by the distance matrix and one or more categorical predictors; and 4) building an induction tree to help interpret how activity sequences change with the predictors. All of these steps are implemented in $\mathrm{R}$ 
with the TraMineR package (Studer, et al., 2011; Gabadinho, Ritschard, Muller, \& Studer, 2011).

\subsubsection{Sequence Representation}

Like all other household activity-travel surveys, the OTAS data is released in a spell format, where each record represents an activity spell or episode of variable duration undertaken by a person, and each person may have more than one activity episode during a day. To conduct sequence alignment, an individual's activity diary first needs to be converted from the spell format to a sequence of letters representing activity states on a fixed time scale. In this study, individual activity diaries are reconstructed with 12 aggregated activity types on 5-min time intervals from 3:00 AM through next day 2:59 AM, so that each activity sequence consists of 288 consecutive activity codes. Figure 1 shows a 'sequence index plot' and a 'state distribution plot' for the transformed activity sequences. In the activity sequence index plot, the first 10 sequences of the subsample are individually rendered with stacked bars depicting the activity states over time. The sequence index plot is useful to visualize individual activity trajectories and the duration spent in each successive activity episode. The activity state distribution plot shows the distribution of activity states at each time interval for all sequences in the subsample (Gabadinho, et al., 2011). Both plots will be used for building an induction tree as the displayed node content. 


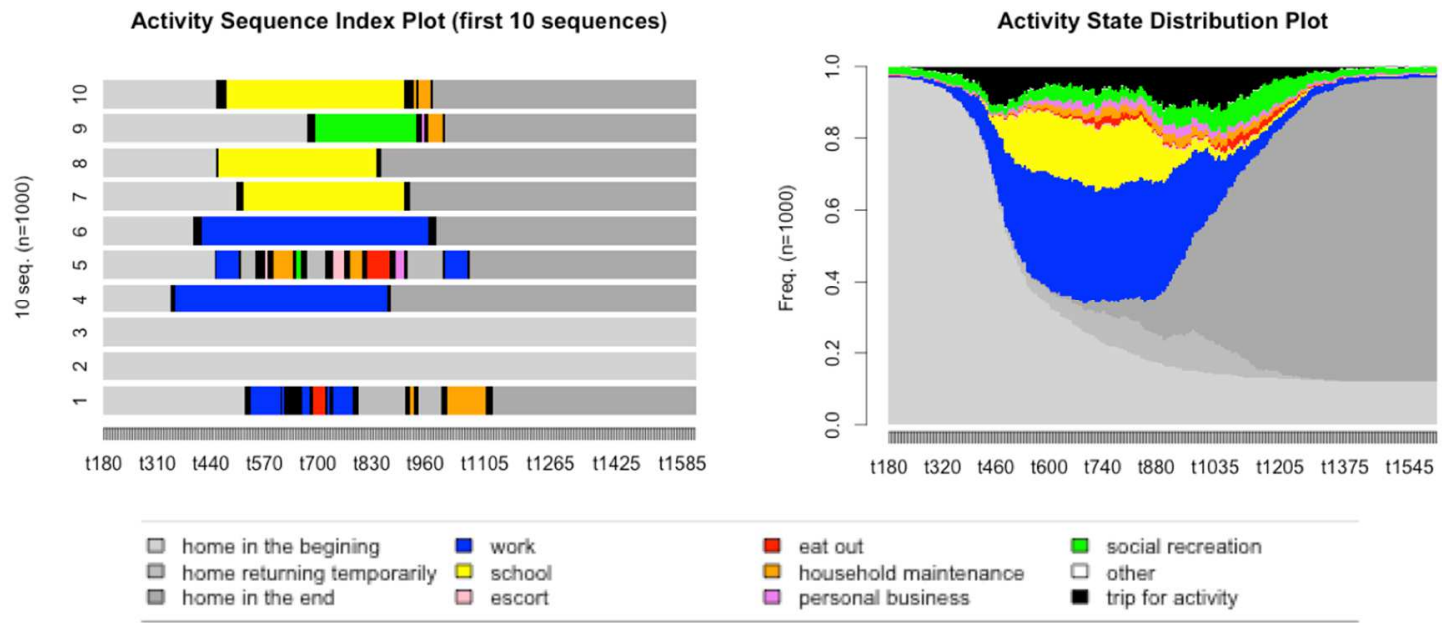

Figure 2-1 Activity Sequence Index Plot and Activity State Distribution Plot

\subsubsection{Sequence Alignment}

Once activity diaries are represented as state sequences with a single attribute of activity type on time intervals of a fixed length, the next step is to align the activity sequences for measuring the dissimilarities between each pair of them. One of the controversial issues of sequence alignment methods in human behavioral applications, as in this study, is how to set up transformation costs or penalties for the two basic operations - substitutions and indels. In fact, existing literature suggests various ways of assigning the transformation costs. For example, the indel cost can be separated into two types - gap opening penalty and gap extension penalty (Wilson, 2001; Shoval \& Isaacson, 2007; Saneinejad \& Roorda, 2009). A higher penalty can be given for gap openings in the first time position of an activity episode than for gap extensions in all the subsequent time positions of that episode. In addition, instead of using a single substitution cost, it is possible to develop a matrix that specifies the substitution costs between all pairs of sequence states (Wilson, 
2001). The substitution cost matrix can also be derived from the probability of transition between sequence states, given that a higher transition rate between two states may indicate a less costly substitution of these states (Lesnard, 2010). However, this study follows the default settings, focusing more on evaluating the validity of sequence discrepancy analysis that is new in transportation research. In other words, the substitution and indel costs are set to 2 and 1, respectively, in this study.

\subsubsection{Discrepancy Analysis of Activity Sequences}

This paper introduces a new methodology for a direct analysis of the association between complex objects described by a distance matrix and one or more categorical variables; there is no need for any prior data reduction technique, such as cluster analysis. This new method is a generalization of MANOVA (Anderson, 2001). The standard MANOVA is concerned with measurable objects that are characterized by multiple continuous dependent variables. On the other hand, the generalized MANOVA can handle complex objects that are not directly measurable, but can be described by a pairwise dissimilarity matrix, such as ecosystems, life trajectories and activity diaries.

The purpose of ANOVA is to test for significant differences among group means by analyzing the variance. Recall that given a certain sample size, the sample variance is a function of the sum of squared deviation from the mean or SS for short. The essence of ANOVA is partitioning the total variance (SST) into two different sources of variance: the within-group variance (SSW) and the among-group variance (SSA). Then, the two variance sources are compared to produce the test statistic of F-ratio. The larger the F- 
ratio value, the more likely it is to reject the null hypothesis that there is no difference among the group means.

For one-way univariate ANOVA, in which a single response variable is linked to one predictor, SSW is the sum of (deviation) squares between individual cases and their group mean, while SSA is the sum of (deviation) squares between group means and the overall sample mean. Next, consider one-way multivariate ANOVA, in which multiple responses are associated with one predictor. Traditionally, MANOVA compares the among-group variance/covariance matrix versus the within-group variance/covariance matrix, instead of the corresponding variances. The covariance here is included because the multiple response variables may be correlated and we need to consider these correlations for the significance test. In case that the correlations between response variables do not really matter, as in independent activity sequence objects, however, we can simply add up the sums of squares across all response variables. Then, we can construct an F-ratio test statistic, as in the univariate ANOVA problem. Such an additive partitioning of the sums of squares in MANOVA can also be thought of geometrically as shown in Figure 1 of Anderson (2001).

The key to generalize the geometric approach of MANOVA to complex objects is based on the fact that "the sum of squared distances between points and their centroid is equal to the sum of squared interpoint distances divided by the number of points" (Anderson, 2001). This relationship has an important implication that an additive partitioning of sums of squares can be obtained without calculating the central locations of groups. For the Euclidean distance measure, the relationship between distances to the 
centroid and interpoint distances was well known early on. It was found that this key relationship holds for any non-Euclidean distance measures equivalently (Anderson, 2001). The importance of this finding is substantial because, unlike the Euclidean distances, the calculation of a central location for non-Euclidean distances, such as a pairwise distance matrix resulting from sequence alignments, is often problematic. Further, Studer et al. (2011) demonstrate that if the distance measure is non-Euclidean, the non-Euclidean distances do not need to be squared before summing them. In short, the new method generalizes the notion of "sum of squares" in ANOVA to non-Euclidean measures of dissimilarity.

Once the test statistics of pseudo F-ratio with any non-Euclidean distance measure is obtained, we need to test the statistical significance. However, we cannot conduct the classical F-test as in the standard ANOVA because the distances between complex objects are not normally distributed and thus the pseudo F-ratio statistic does not follow a Fisher distribution under the null hypothesis. Instead, we need to consider a permutation test in order to obtain a new distribution of the pseudo F-ratio under the null hypothesis. The permutation test works as follows. First, the complex objects are exchanged among the different groups of a categorical predictor through a random permutation. Second, a new pseudo F-ratio statistic, called $F_{\text {permuted, }}$, is computed. Third, the first and second steps are repeated for all possible permutations, which give the entire distribution of the pseudo F-ratio statistic under the true null hypothesis. Fourth, from this distribution, the p-value of the observed pseudo F-ratio statistic ( $F_{\text {observed }}$ ) is assessed by evaluating the proportion of $F_{\text {permuted }}$ that are higher than $F_{\text {observed. }}$ Since the number of all possible permutations is 
often huge, it is usually practical to perform 1,000 permutations for tests with a $5 \%$ significance level (Studer, et al., 2011; Anderson, 2001). Table 3 compares standard ANOVA versus generalized MANOVA in one-way design with respect to the calculation of a test statistic and the significance test.

Table 2-3 Standard ANOVA vs. Generalized MANOVA: One-Way Design

\begin{tabular}{|c|c|c|}
\hline & Standard ANOVA & Generalized MANOVA \\
\hline \multirow{5}{*}{$\begin{array}{c}\text { Test } \\
\text { Statistic }\end{array}$} & $F-$ ratio $=\frac{S S_{A} /(a-1)}{S S_{W} /(n-a)}$ & pseudo $F-$ ratio $=\frac{S S_{A}^{*} /(a-1)}{S S_{W}^{*} /\left(n^{*}-a\right)}$ \\
\hline & - $\quad S S_{T}=S S_{W}+S S_{A}$ & - $\quad S S_{T}^{*}=S S_{W}^{*}+S S_{A}^{*}$ \\
\hline & $\begin{array}{l}\text { - } S S_{T} \text { is the sum of squared Euclidean } \\
\text { distances from individuals to the grand } \\
\text { centroid }\end{array}$ & $\begin{array}{l}\text { - } S S_{T}^{*} \text { is the sum of all pairwise } \\
\text { distances divided by the number of } \\
\text { objects }\end{array}$ \\
\hline & $\begin{array}{l}S S_{W} \text { is the sum of squared Euclidean } \\
\text { distances from individuals to their } \\
\text { group centroid }\end{array}$ & $\begin{array}{l}\text { - } S S_{W}^{*} \text { is the sum of all pairwise } \\
\text { distances within groups divided by } \\
\text { the number of objects }\end{array}$ \\
\hline & $\begin{array}{l}-S S_{A} \text { is the sum of squared Euclidean } \\
\text { distances from group centroids and the } \\
\text { grand centroid }\end{array}$ & - $\quad S S_{A}^{*}=S S_{T}^{*}-S S_{W}^{*}$ \\
\hline $\mathrm{p}$-value & F test & permutation test \\
\hline
\end{tabular}

Note: (1) $a$ refers to the number of levels or groups of a covariate; (2) in the generalized MANOVA, $n^{*}=n$ $(n-1) / 2$ where $n$ is the sample size.

In the above, the one-way design of discrepancy analysis was discussed; that is, a single factor is associated with a distance matrix of the complex sequence objects. The one-way design can be nicely extended to a multi-way design in which multiple factors are involved. For more information on formula of SST, SSW, and SSA to compute a pseudo F test statistic in the multi-way design, refer to McArdle and Anderson (2001). As in the one-way discrepancy analysis, since the F distribution is not suitable for evaluating 
the pseudo F-ratio statistic, we consider the permutation test again. This paper conducts the multi-way discrepancy analysis to simultaneously find out multiple factors that explain discrepancies among individuals' activity patterns. Since those factors are often highly correlated, their unique effects after controlling for other effects are more appropriate than their marginal effects obtained from a series of the one-way discrepancy analysis.

\subsubsection{Tree-Structured Analysis of Sequences}

Indeed the sequence discrepancy analysis with either a single factor or multiple factors can explain which variables have significant effects on the discrepancy among activity sequences. However, it is hard to tell what the effects are, namely, how activity sequences may vary with the value of the predictors. To complement this limitation, an induction tree is built. In general, trees work as follows. First, all sequence objects are located in an initial node. Then, each node is recursively partitioned by the value of a predictor. The predictor and the split are determined so that the resulting child nodes are different from one another as much as possible. The procedure is repeated at every new node until certain stopping criteria are met. As building a tree with state sequences is very rare in existing literature, however, this study follows the instructions suggested by Studer et al. (2011). Their tree is slightly different than popular tree algorithms, such as CHAID, in several aspects. First, while CHAID can only handle a categorical variable, the proposed tree is built on the basis of sequence objects that are neither continuous nor categorical. Second, the proposed tree is binary in that each node is split into only two 
subsamples, unlike multi-branch trees of CHAID. Third, a pseudo R2 derived from the one-way discrepancy analysis is used as a node splitting criterion. In other words, each node is split with the predictor and its value achieving the highest pseudo R2 value. Fourth, the significance of the one-way pseudo F-ratio that is determined through permutation tests is used as a stopping criterion. At each node, the tree stops growing a branch once the selected split encounters a non-significant $\mathrm{F}$ value.

\subsection{Results and Discussions}

\subsubsection{Sequence Discrepancy Analysis with Multiple Factors}

Table 4 shows the results of the multi-factor discrepancy analysis of activity sequences characterized by a pairwise sequence alignment distance matrix. For illustrative purpose, only seven significant covariates were selected, including one interaction term. The set of covariates explained approximately $19.4 \%$ of the total discrepancy among the daily activity sequences of 1,000 persons since the global pseudo $\mathrm{R}^{2}=0.194$. The overall model was statistically significant, indicated by the global pseudo F value of 34.064 and $\mathrm{p}<.05$. The most significant factor was an indicator of whether or not the person was a K-12 student. If the K-12 indicator was removed, the global pseudo $\mathrm{R}^{2}$ decreased by 0.046. This difference was significant since the pseudo $\mathrm{F}$ value for that indicator was 57.034 , which was a value attained less than five times out of the thousand permutations. The worker indicator was also significant. Removing the indicator variable from the model reduced the global pseudo $\mathrm{R}^{2}$ by 0.043 , which was significant since the pseudo $F_{\text {worker }}=52.904$ was attained less than five times amongst the thousand permutations. As 
for the other indicator variables, results indicated that full-time college students, persons with driver's license, adults over age 65 made moderate but significant contributions to explain the total discrepancy of activity sequences. There also existed statistically significant discrepancy in activity sequences among five groups of different household size $(1,2,3,4$, and 5+). Finally, the sequence discrepancy of activity diaries were strongly influenced by an interaction of the Worker and Adult-over-age-65 covariates.

Table 2-4 Multi-Factor Discrepancy Analysis

\begin{tabular}{|c|c|c|c|c|}
\hline Variable & Variable Type & $\begin{array}{c}\text { Pseudo F } \\
\text { (for each variable) }\end{array}$ & $\begin{array}{c}\Delta \text { Pseudo R } \\
\text { (for each variable) }\end{array}$ & p-value \\
\hline Worker & indicator & 52.904 & 0.043 & 0.001 \\
K-12 student & indicator & 57.034 & 0.046 & 0.001 \\
Full-time college student & indicator & 2.829 & 0.002 & 0.008 \\
Licensed & indicator & 4.658 & 0.004 & 0.001 \\
Adult over age 65 & indicator & 3.220 & 0.003 & 0.003 \\
Household size & 5 categories & 2.361 & 0.002 & 0.017 \\
Worker* Adult over age & interaction & 2.337 & 0.002 & 0.027 \\
\hline 65 & & $\begin{array}{c}\text { Pseudo F } \\
\text { (for total) }\end{array}$ & $\begin{array}{c}\text { Pseudo R } \\
\text { (for total) }\end{array}$ & p-value \\
\cline { 3 - 5 } & & 34.064 & 0.194 & 0.001 \\
\hline
\end{tabular}

\subsubsection{Tree Analysis of Activity Sequences}

Using the same set of covariates as in the previous multi-factor discrepancy analysis, but without the interaction term, an induction tree was built for the subsample of 1,000 activity trajectories, which is shown in Figure 2. To display more comprehensive information about the content at each node, the same tree was built with both activity sequence index plots and activity state distribution plots in the top and bottom panels of 
Figure 2, respectively. In addition to the node content, other important information is displayed on each node, including node size $(n)$ and within-node discrepancy $(s 2)$. Moreover, a selected split covariate and its associated one-way pseudo $\mathrm{R}^{2}$ are shown at the bottom of each parent node. The selected binary split of a covariate is indicated at the top of each child node.

The global pseudo $\mathrm{R}^{2}$ of the tree was $18.96 \%$, which is slightly lower than that of the multi-factor discrepancy analysis in Table 4. However, it should be noticed that while in the discrepancy analysis the seven covariates including one interaction term turned out to be significant, only four covariates of them were involved for developing the tree to produce the similar pseudo $\mathrm{R}^{2}$ value. This might be because several interaction effects are automatically detected on the tree. For example, it was found that household size had more influences on the activity sequences of non-workers who do not go to K-12 school (e.g., homemaker) than those of K-12 students. As for workers, household size mostly influenced the daily activity patterns of younger workers (less than age 65), not older workers (over age 65).

In addition to the automatic detection of interaction effects and the provision of a comprehensive view of the sequence-covariate link, the induction tree yielded seven clusters at the terminal nodes. As shown in the tree with state distribution plots, it is possible to discover the differences among the seven clusters in the distribution of activity types at each 5-min time point. For example, see the terminal node indicating K12 students. Most of them conducted the 'school' activity (yellow) in the midday and their 'trip' activity (black) was noticeably peaked at two time points. In addition, non- 
workers who are not a K-12 student were separated by household size. Those with small household size $($ h_size $<=2$ ) spent more time at home (grey) than those with large household size (h_size $>2$ ). Further, one cluster of older workers was discovered, which differs from the other three clusters of younger workers. Compared to younger workers, older workers spent less time on working (blue) and social recreation (green) and more time on personal business (violet), such as health care. Not surprisingly, the 'trip' activity of older workers was spread over the midday without any clear peak time points. 
(a) Tree with activity sequence index plots

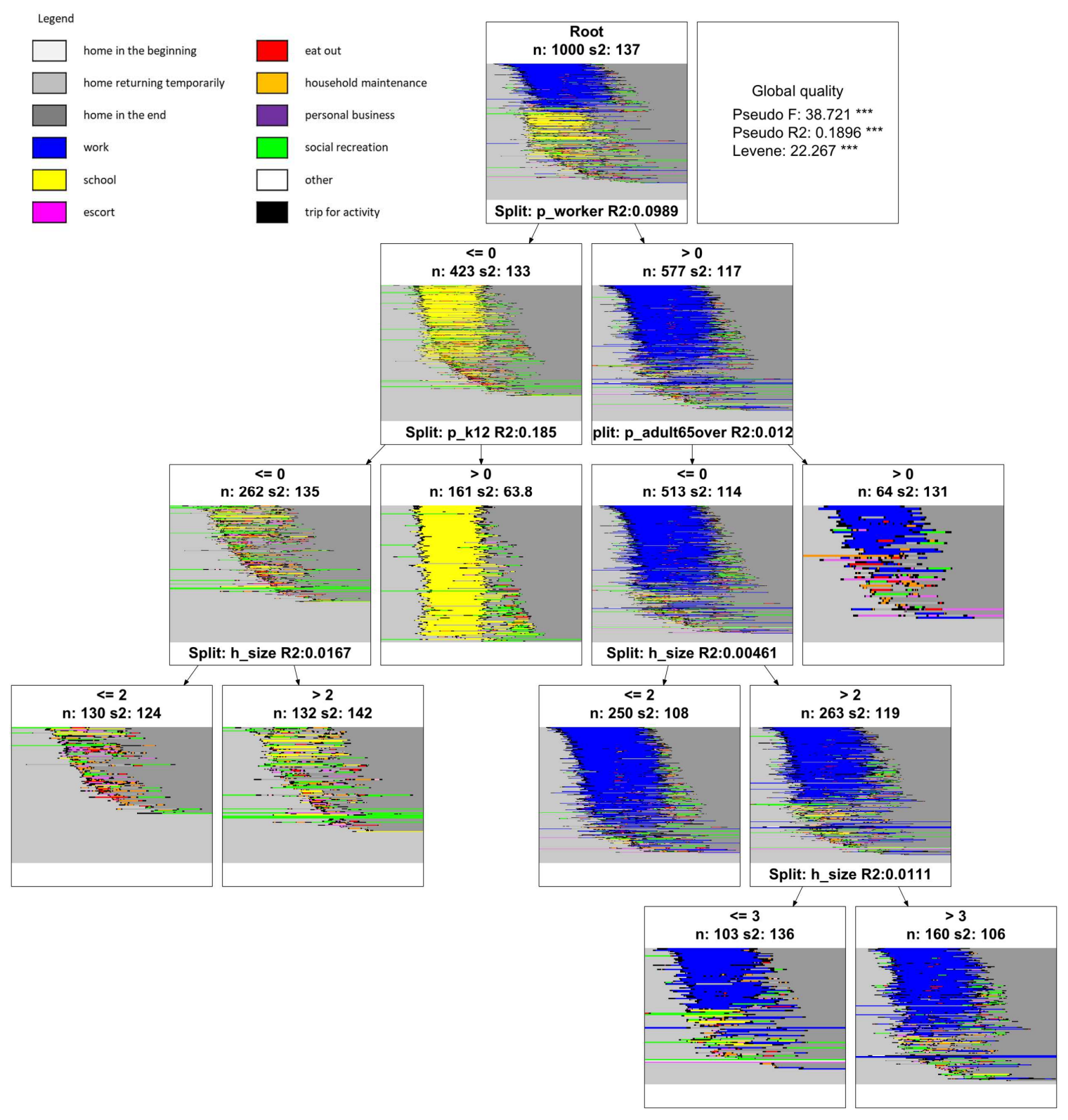


(b) Tree with Activity State Distribution Plots

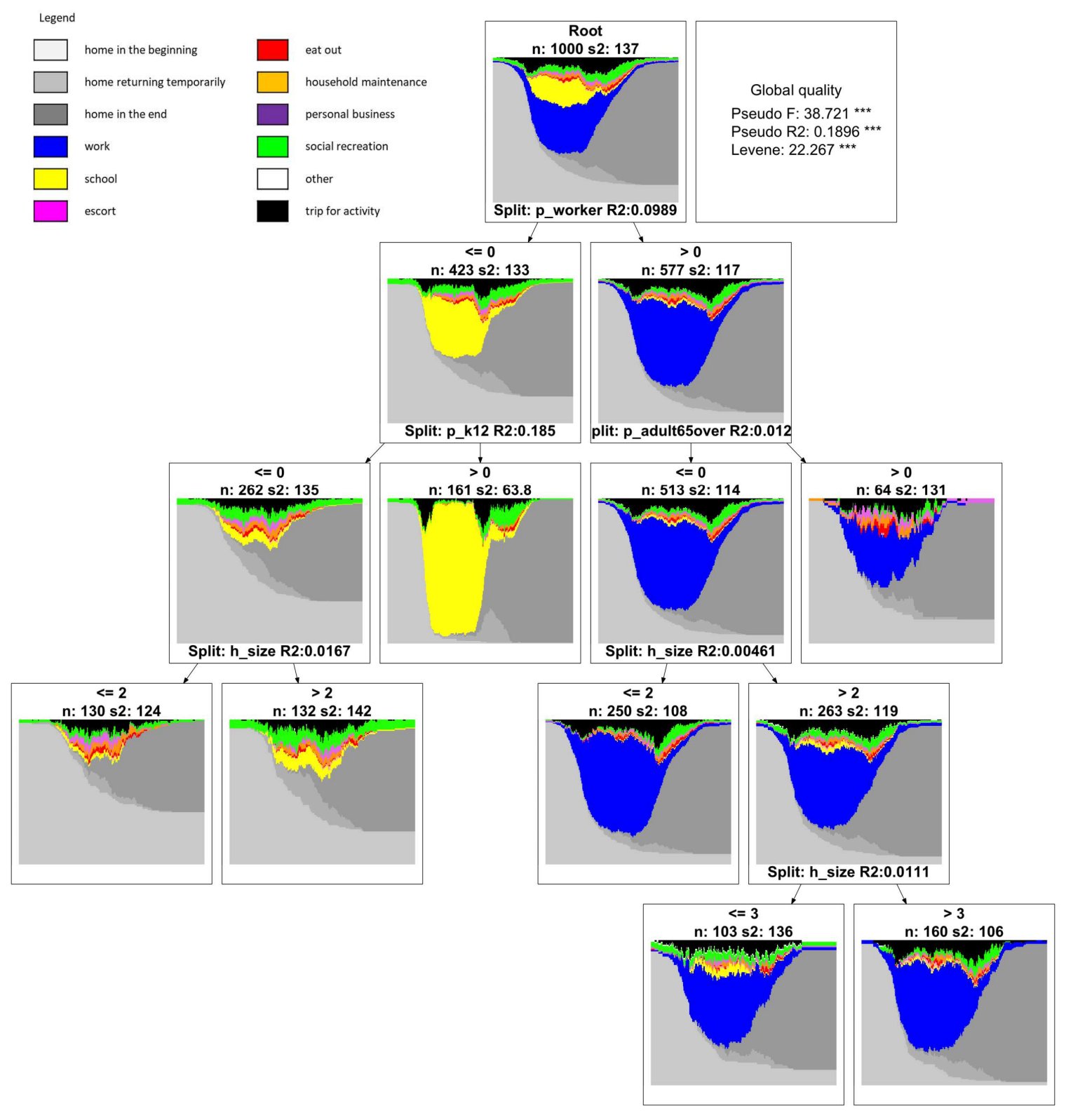

Figure 2-2 Induction Trees of Activity Sequences:

(a) with activity sequence index plots and (b) with activity state distribution plots 


\subsection{Conclusion}

Individuals' daily activity-travel patterns are complex due to interactions of numerous aspects embedded in them. An "old" question in activity-based travel behavior analysis is what explains the complexity of activity-travel patterns. To answer the question, this study proposed the discrepancy analysis of activity sequences. Viewing individual activity patterns as holistic and sequential objects, activity diaries were first converted into sequences of characters representing activity states. Then, the total discrepancy in the activity sequences was defined with a pairwise dissimilarity matrix between all sequences that was obtained from sequence alignment methods. Following the principle of ANOVA, the total sequence discrepancy was partitioned into explained among-groups discrepancy and residual within-groups discrepancy. This partition enabled to measure the strength of the association between activity sequences and covariates by calculating a pseudo $\mathrm{R}^{2}$ and to assess the statistical significance of the association through the permutation tests of a pseudo F-ratio value. In addition to the sequence discrepancy analysis, this study developed an induction tree to help understand how individual activity sequences vary with the influential covariates.

Most of the existing applications of sequence alignment to activity-travel diary data have been restricted to calculating and classifying the dissimilarities of activity sequences, missing useful knowledge on activity sequences. It is expected that this research will allow us to explore the unknown area. In addition, this study would make a practical contribution to a micro-simulation framework for activity-based travel demand modeling. Some activity-based models assume a sequential scheduling process in which 
individuals decide what to do next at every time point. The approach is often criticized for the absence of any pre-planning process where activities are scheduled first on the basis of their priority and the schedule is implemented next. Gliebe and Kim (2010) respond to this criticism by assigning household roles to individuals before simulating them. This market segmentation can create a propensity for a certain type of behavior from which a wide range of activity-travel patterns may emerge. However, the market segmentation does not really capture the sequential decision process in advance.

Discrepancy analysis with sequence alignment proposed in this paper may enable a more suitable segmentation for agents who are simulated through a sequential scheduling process.

This study certainly leaves room to be improved in several aspects. First of all, sequence alignment methods combined with discrepancy analysis in this paper can be further fine-tuned with different transformation cost settings for indels and substitutions. In addition, activity sequences can be represented in a different way by considering multiple activity attributes simultaneously, such as activity location, travel mode and time, the presence of persons, etc. Lastly, it is worthwhile to compare the cluster solutions discovered by building a tree with those obtained from a classical cluster analysis following sequence alignment methods. Thus, it may be possible to empirically verify whether or not the new methodological combination presented in this paper will truly overcome information loss caused by the previous popular cluster-based approach. 


\section{DISCRETE-TIME ACTIVITY DURATION ANALYSIS}

Most existing activity-based travel demand models are typically implemented on a tourbased microsimulation framework (Davidson et al., 2007; Vovsha, Bradley, \& Bowman, 2005). On the tour-based framework, the basic unit of analysis for modeling travel is a tour, not a trip. In the context of travel demand models a trip represents a travel unit connecting two locations, while a tour is defined as a chain of trips starting and ending at home or work (Donnelly et al., 2010). The main advantages of the tour-based structure are to preserve a consistency among multiple trips within a tour in terms of travel mode, destination, and timing. On the microsimulation framework, a full list of households and persons in the synthetic population is simulated during the course of a day. Compared with the "zonal enumeration" approach that is usually used to implement the conventional trip-based four-step models, the microsimulation approach has several advantages (Donnelly et al., 2010). First, the microsimulation may resolve several critical biases that result from demographic, spatial, and temporal aggregations. Second, the microsimulation models are computationally more efficient, virtually allowing an unlimited number of predictors. Third, the microsimulation outcomes look more realistic, being similar to individual activity-travel diaries in travel survey data. Lastly, the microsimulation models are better integrated with the state-of-the-art transport network analysis tools, such as Dynamic Traffic Assignment (DTA), by providing trip tables or individual trip schedules at a level of compatible temporal resolution (e.g., 30 or 15 minute). 
Two different approaches are available to describe people's daily decisionmaking processes on activity and travel in the tour-based microsimulation framework (Gliebe \& Kim, 2010). For the first approach, it is assumed that people preplan the number of tours for a day and the number of trips within the tour(s), set the duration of activities, and then calculate the remaining time windows under their space-time constraints. If things do not fit well, they re-schedule the day by adjusting activity timing, activity locations and/or travel modes. This approach is sometimes referred to as "preplanning" or "rubber-banding" scheduling. The second approach assumes that as the day goes by, people decide what to do next, where to do it, and how to get there. These sequential decisions depend on previous activities, time windows, business hours, intrahousehold interactions, and so on. This approach is also known as "sequential" or "growing” scheduling.

The pre-planning scheduling microsimulation is a popular choice because of the plausible assumption that individuals' daily activities are planned in advance, following a fixed hierarchy of activity types. The activity hierarchy typically contains solo mandatory, joint maintenance, joint discretionary, allocated maintenance, and solo discretionary activities, in order from most important to least important. However, there are little empirical evidences for such a rigid structure of activity priorities (Doherty \& Mohammadian, 2011). The authors demonstrated that more than $50 \%$ of mandatory activities are not planned in advance in forming home-based tours. On the other hand, the sequential scheduling microsimulation is more flexible because there is no need to predetermine activity priorities and trip/tour frequencies. Instead, this approach focuses more 
on how individuals' activity-travel decisions change over time (Gliebe \& Kim, 2010). The authors made a novel proposition that "utility for daily activity-travel alternatives is updated rather than accumulated."

It is worthwhile to mention that the growing scheduling approach accounts for the activity pre-planning behavior as well, but in a different way. Instead of predicting individuals' number of tours and stops a priori, a very specific household role can be assigned to each household member in the beginning of the simulation day to capture idiosyncratic patterns of pre-planning behavior. The household roles include, for example, working outside home with childcare responsibilities, working outside home while attending college classes, working outside home with planned joint activities with other household adults, and so on (Gliebe \& Kim, 2010). Alternatively, sequence alignment methods can be applied to segment people into similar groups in terms of their activity sequences (Kim, 2014). Activity diaries are first transformed into sequences of characters representing activity types on fixed time intervals, say, 5-min time intervals. Then, the dissimilarities between all activity sequences are measured through sequence alignment. The resultant pairwise dissimilarity matrix is combined with ANOVA-like analysis to find out significant covariates affecting variations in the activity sequence patterns. An induction tree is also introduced to display how activity sequences vary with the covariates.

Such a sequential scheduling approach requires a series of linked dynamic discrete choices of activity episodes, locations, and travel modes to incrementally build an entire day's activity-travel patterns for individuals in households (Gliebe \& Kim, 
2010). In other words, every 5 or 10 minute an individual decides whether to stay in a current activity or move to a next activity (next activity choice), and then once a new activity is chosen, the individual determines where to do it (next location choice) and how to get there (next mode choice). As for the next activity choice, one may think of a typical discrete choice model, such as multinomial logit regression. However, the decision on next activity choice is strongly time-dependent. For example, it depends on time spent in a current activity, cumulative time spent in all the previous activities for the day, and time of day. Static discrete choice models can only implicitly account for such time dependencies (Vovsha \& Bradley, 2004). A promising statistical method to explicitly incorporate such time dependencies into the next activity choice is either hazard-based duration models or dynamic discrete choice models (Ettema, Borgers, \& Timmermans, 1995). In this paper we introduce the simplest form of dynamic discrete choice models, which is essentially the same as hazard-based duration models in a discrete time framework (Heckman \& Navarro, 2007).

Hazard-based duration models deals with time to an event, using a hazard function that represents the conditional probability of an event occurring at a time period, given that the event did not occur before the time period. Duration analysis is often referred to as event history analysis in social science, survival analysis in medical science, and failure time analysis in industrial engineering. There is a wide range of hazard-based duration models. One broad distinction between the duration models can be made by whether duration times are measured in continuous or discrete time (Steele, 2005). Most existing duration models in transportation research belong to the continuous- 
time approach. Bhat and Pinjari (2008) list the recent applications of the continuous-time duration models to activity participation and scheduling studies.

Although activity duration data are continuous in nature, we devote ourselves to the discrete-time approach in this study for several reasons. First, activity diaries are retrospectively collected from household travel surveys. Therefore, respondents are more likely to approximate their activity arrival and departure times to multiples of 5 minutes (e.g., 5, 10, 15, 30, 60 minutes, etc.). Bhat (1996) suggests that activity duration data should be treated as being discrete. Second, as activity durations are discretely measured, the data may contain numerous ties at those discrete time intervals. While discrete-time models can easily handle the tied observations, serious biases can occur in the use of Cox proportional hazards model that is one of the most popular continuous-time models (Steele, Diamond, \& Wang, 1996). In the context of activity-based modeling, such tied observations are common when considering joint activity participations of household members. Third, it is also more straightforward to include time-varying covariates into a discrete-time model than into a continuous-time model (Steele, 2005). The status of household members, traffic path information provided by DTA, and transit operating hours are varying over time, which are important variables in advanced activity-based models, even if they are not considered in this study. Lastly, discrete-time duration models can be relatively easily extended to recurrent events, competing risks, and multiple states, compared with continuous-time duration models.

The aim of this paper is to introduce a discrete-time duration model that can be designed for all of these complex situations and then illustrate its application to the 
analysis of next activity choice and activity duration. In the next section we will review the discrete-time duration analysis. Then, we describe data used for this study, together with data transformation required to perform the discrete-time method and our model development process. Next, the model estimation results will be summarized for both random and fixed effects. In conclusion, some limitations of this study will be presented.

\subsection{Background}

Since discrete-time hazard-based duration models are rare in transportation research, in this section we briefly overview the methodology based on Steele's multiple works (Steele, Goldstein, \& Browne, 2004; Steele, 2005; Steele, 2008; Steele, 2011). For more detailed information, an excellent textbook is available (Singer \& Willett, 2003). We begin the overview with a simplest case in which a single non-recurrent event is concerned. Then we add other complexities associated with recurrent events, competing risks, and multiple states in order.

\subsubsection{A Discrete-Time Duration Model for a Single Event}

Suppose that for each episode $i$, we observe duration $y_{i}$ accounting for time to a single target event (e.g., leaving a current activity episode). Suppose also that the duration $y_{i}$ is measured in discrete time intervals indexed by $t(t=1,2,3, \ldots, K)$, which is either fully observed if the event occurs $\left(\delta_{i}=1\right)$ or right-censored if not $\left(\delta_{i}=0\right)$. The first step of a discrete-time analysis is to convert the individual-episode format to an individual- 
episode-period format; for each time period $t$, we define a binary response $\mathrm{y}_{\mathrm{ti}}$ that indicates whether or not the event occurred during the time interval as follows:

$$
y_{t i}= \begin{cases}0 & t<y_{i} \\ 0 & t=y_{i}, \delta_{i}=0 \\ 1 & t=y_{i}, \delta_{i}=1\end{cases}
$$

In the individual-episode file, for example, $y_{i}=4$ means that an episode $i$ experiences an event during the fourth time interval. In the individual-episode-period format, the time to an event or the duration is converted into the four binary responses, namely, $\left(y_{1 i}, y_{2 i}, y_{3 i}, y_{4 i}\right)=(0,0,0,1)$. If the episode is right-censored at the same time interval, then the binary responses are coded as $\left(y_{1 i}, y_{2 i}, y_{3 i}, y_{4 i}\right)=(0,0,0,0)$.

Now we define a discrete-time hazard for time interval $t$, that represents the conditional probability of an event occurring during interval $t$, given that the event did not occur before $t$, as follows:

$$
h_{t i}=\operatorname{Pr}\left(y_{t i}=1 \mid y_{t^{\prime} i}=0 \text { for } t^{\prime}<t\right)
$$

The next step is to model how the discrete-time hazard function depends on duration and covariates. Note that in the transformed data set (i.e., the individual-episodeperiod format), the dependent variable of interest is binary, indicating the occurrence of an event. A popular solution to analyze binary responses is to perform a logit transformation of the hazard function. As a result, the log odds of the discrete-time 
hazard is modeled as a linear combination of two sets of predictors, which is given as follows:

$$
\operatorname{logit}\left(h_{t i}\right)=\log \left(\frac{h_{t i}}{1-h_{t i}}\right)=\alpha(t)+\boldsymbol{\beta}^{\prime} \boldsymbol{X}_{t i} \cdots \cdots \cdots
$$

where $\alpha(t)$ is a function of time period $t$ to incorporate the duration effect, namely, the dependence of the hazard function on $t$, which is referred to as the baseline logit-hazard. There are two different ways of specifying the baseline logit-hazard: non-parametric and parametric. The non-parametric specification includes a sequence of temporal dummy variables. Since there are $K$ time intervals in the transformed data set, the baseline logithazards can be specified with the $K$ temporal dummies, $\alpha(t)=\alpha_{1} D_{1}+\alpha_{2} D_{2}+\cdots+$ $\alpha_{K} D_{K}$. The resultant multiple intercepts represent the baseline logit-hazard for each time period. Although this non-parametric approach is attractive because of its flexibility, there is a practical drawback. In case that the number of time periods in a data set is large, the model needs a substantially large number of dummy variables, which is unwieldy. To be more parsimonious, one can parameterize the duration effect. Depending on a plot of the observed hazards over time, a variety of forms of $\alpha(t)$ are possible, such as a linear function $\alpha(t)=\alpha_{0}+\alpha_{1} t$, a quadratic form $\alpha(t)=\alpha_{0}+\alpha_{1} t+\alpha_{2} t^{2}$ or a log function $\alpha(t)=\alpha_{0}+\alpha_{1} \log (t)$, where $\alpha_{0}$ represents an overall intercept term.

On the other hand, in Equation (1), $\boldsymbol{X}_{t i}$ is a set of covariates to detect observed heterogeneity in hazard across episodes. The covariates are either time constant or time varying. In discrete-time models, it is straightforward to add time-varying covariates by 
placing their different values in time periods. In addition, the assumption of proportionality that the effects of covariates are constant over time is common in continuous-time models. In discrete-time models, however, the proportionality assumption can be easily relaxed by introducing the interactions between $x$ and $t$ as an additional explanatory variable.

\subsubsection{A Discrete-Time Duration Model for Recurrent Events}

An event may occur one or more times to an individual during a given observation period. For example, in activity survey data, most individuals participate in more than one out-of-home activity during a day. In other words, an individual experiences an event that terminates an activity to carry out another activity several times for a day. In a discrete-time data set with recurrent events, we can define a series of binary response $\left\{y_{t i j}\right\}$ in which $y_{t i j}$ indicates whether an event has occurred in time interval $t$ for episode $i$ of individual $j$. Then, the corresponding discrete-time hazard function can be written as

$$
h_{t i j}=\operatorname{Pr}\left(y_{t i j}=1 \mid y_{t^{\prime} i j}=0 \text { for } t^{\prime}<t\right)
$$

If recurrent events are observed to an individual, it cannot be assumed that the durations of episodes are independent within the same individual. There may be unobserved heterogeneity, also known as shared frailty, between the individuals. Such unobservables can be captured using multilevel modeling techniques. Note that recurrent events occur in a two-level hierarchical structure where episodes in the lower level are nested within 
individuals in the upper level. Hence, in discrete-time analysis, we can add a random intercept term to Equation 1, which is written as

$$
\operatorname{logit}\left(h_{t i j}\right)=\log \left(\frac{h_{t i j}}{1-h_{t i j}}\right)=\alpha(t)+\boldsymbol{\beta}^{\prime} \boldsymbol{X}_{t i j}+u_{j} \cdots \cdots \ldots
$$

where $u_{j}$ is a random effect associated with the $j$ th individual. The values of $u_{j}$ are typically assumed to follow a normal distribution with zero mean and variance $\sigma_{u}^{2}$. In such a two-level random intercept model, the log-odds of an event in interval $t$ is only shifted by $u_{j}$ for the $j$ th individual. In a more complex random coefficient model, the coefficients $\beta$ s can be specified to be random across individuals.

\subsubsection{Modeling Competing Risks and Multiple States}

Another common extension of duration models is to take into account more than one kind of event. So far, it is assumed that a single type of event occurs to an individual. However, in many situations, multiple types of event, also referred to as competing risks, may be competing to end an episode. If competing risks arise, the dependent variable in the discrete-time data is no longer binary, and it becomes multinomial. Therefore, Equation 2 can be generalized to a multinomial logit model with a random intercept.

Suppose that there are $R$ types of event. In competing-risks analysis, a categorical response $y_{t i j}$ is defined for each interval $t$ of episode $i$ of individual $j$. If an event of type $r$ occurs in interval $t$, then $y_{t i j}=r$ for $r=1, \ldots, R$, while if no event occurs, $y_{t i j}=0$. The event-specific discrete-time hazards are defined as follows: 


$$
h_{t i j}^{(r)}=\operatorname{Pr}\left(y_{t i j}=r \mid y_{t^{\prime} i j}=0 \text { for } t^{\prime}<t \text { and } r=1, \ldots, R\right)
$$

and Equation 2 for the single event analysis becomes

$$
\operatorname{logit}\left(h_{t i j}^{(r)}\right)=\log \left(\frac{h_{t i j}^{(r)}}{h_{t i j}^{(0)}}\right)=\alpha^{(r)}(t)+\boldsymbol{\beta}^{(r) \prime} \boldsymbol{X}_{t i j}^{(r)}+u_{j}^{(r)}, r=1, \ldots, R
$$

The above random intercept multinomial logit model consists of $R$ equations contrasting the risk of an event of type $r$ with the risk of no event as the reference category. The form of baseline logit-hazard and the set of covariates may be specified differently for each type of event. In addition, the effects of duration and covariates may vary across event types. Further, the random effect associated with individual $j$ may vary by event type, even if the random effects (i.e., $u_{j}^{(1)}, u_{j}^{(2)}, \ldots, u_{j}^{(R)}$ ) are assumed to follow a multivariate normal distribution with a covariance matrix.

In competing-risk models, we focus on transitions from one origin state. However, there may be multiple origin states from which multiple types of event are competing to end the origin state. A simple way of handling multiple origin states simultaneously is to add dummy variables indicating which state is occupied during interval $t$ to the competing-risk model of Equation 3 as predictor variables. A more general discrete-time duration model for recurrent events, competing risks, and multiple states is described by Steel et al. (2004). 


\subsection{Application to Activity Duration Analysis}

The main goal of this study is to develop a hazard-based duration model on a discretetime framework for daily in-home and out-of-home activity episodes. In this study, an activity episode is defined as a continuous period during which an individual is at risk of experiencing an event that terminates an origin or current activity and then moves to a destination or next activity. The duration (i.e., the time to an event) of activity episode is measured in discrete time intervals. This study takes into account complex situations as we consider multiple states for the origin activity type as well as competing risks for the destination activity. An additional complexity comes from that activity episodes are recurrent within individuals. In this section, we describe the sample data and their transformation, and the model development.

\subsubsection{Sample Data Definition}

In this study we use the 2011 Oregon Travel and Activity Survey (OTAS). The Portland metropolitan portion of the 2011 OTAS records daily in-home and out-of-home activities of about 11,000 persons of nearly 4,800 households. Among them, we select those aged 65 and over who do not work in order to reduce computational burden. Another important reason to study this particular group of people is that elderly travel behavior is an interesting research topic among transportation planners as the Baby Boomers just started retiring a few years ago. For simplicity, we also remove persons with any type of censored observations, including left-censored observations (i.e., out-of-home activities in the beginning of the day), right-censored observations (i.e., out-of-home activities in 
the end of the day), left-right-censored observations (i.e., stay at home or travel all day). In short, this study examines only retirees who participated in at least one out-of-home activity, starting and ending the day at home. Table 1 compares the frequency and average duration of aggregated activity types between all persons and retirees. Note that in-home activities are subdivided into three levels: home in the beginning of the day, home returning temporarily in the middle of the day, and home in the end of the day. As expected, retirees stayed longer at home than all persons (430 vs. 344 min.). In addition, retirees' average duration minute of all out-of-home activities was one half of that of all persons (53 vs. $125 \mathrm{~min}$.). When excluding subsistence activity types, such as work, work-related, and school activities, however, the average durations for each type of outof-home activity were similar each other between retirees and all persons. 
Table 3-1 Frequency and Average Duration of Activity Episodes for Retirees

\begin{tabular}{|c|c|c|c|c|}
\hline \multirow[b]{2}{*}{ Aggregate Activity Type } & \multicolumn{2}{|c|}{$\begin{array}{l}\text { All Persons } \\
(n=9,339)\end{array}$} & \multicolumn{2}{|c|}{$\begin{array}{l}\text { Retirees } \\
(n=586)\end{array}$} \\
\hline & Frequency & $\begin{array}{l}\text { Average } \\
\text { Duration } \\
\text { (in min.) }\end{array}$ & Frequency & $\begin{array}{l}\text { Average } \\
\text { Duration } \\
\text { (in min.) }\end{array}$ \\
\hline \multicolumn{5}{|l|}{ In-home Activity } \\
\hline home in the beginning & 9,339 & 358.7 & 586 & 476.5 \\
\hline home in the middle & 4,630 & 116.7 & 270 & 142.0 \\
\hline home in the end & 9,339 & 555.3 & 586 & 671.4 \\
\hline Sub-total & 23,308 & 343.5 & 1,442 & 430.0 \\
\hline \multicolumn{5}{|l|}{ Out-of-home Activity } \\
\hline work & 5,012 & 368.6 & & \\
\hline work-related & 1,509 & 104.0 & & \\
\hline school & 2,224 & 368.1 & & \\
\hline eat out & 2,295 & 47.6 & 195 & 62.6 \\
\hline escort & 3,115 & 8.8 & 107 & 8.7 \\
\hline health care & 790 & 69.6 & 105 & 66.9 \\
\hline personal business & 2,820 & 31.0 & 370 & 33.8 \\
\hline shopping & 4,087 & 30.5 & 488 & 37.7 \\
\hline social recreation & 4,072 & 97.2 & 348 & 108.7 \\
\hline Sub-total & 25,924 & 125.0 & 1,613 & 53.0 \\
\hline
\end{tabular}

\subsubsection{Data Transformation}

The original survey data set is released in an individual-episode file format where a twolevel hierarchical structure is revealed with activity episodes (lower level) nested within individuals (upper level). The key attributes of activity episodes are obtained from the survey data, including origin activity type, destination activity type, activity duration, activity location, travel mode, and so on. The dependent variable of this study is the duration of activity episode, which is defined as time to an event terminating an origin activity state. Although the duration variable is continuous in nature, in this study it is 
treated as an interval variable because more than $50 \%$ of the duration times are recorded to the nearest multiples of 5 (e.g., 5, 10, 15, $30 \mathrm{~min}$, etc.). On the other hand, the activity diaries involve both the multiple states of origin activity and the competing risks of destination activity. Table 2 shows the transitions of activity episodes from an origin activity to a destination activity. It is important to note that different destination types are allowed for each origin state. For example, it is not allowed to move from an in-home origin (i.e., H1 and H2) to an in-home destination (i.e., H2 and H3). For all the out-ofhome origin activity types, it is allowed to move to any type of destination, including the same activity type.

Table 3-2 Transitions of 2,469 Activity Episodes from Origin to Destination

\begin{tabular}{|c|c|c|c|c|c|c|c|c|c|c|}
\hline \multirow{2}{*}{\multicolumn{2}{|c|}{ From $\backslash$ To }} & \multicolumn{9}{|c|}{ Destination Activity (Competing Risks) } \\
\hline & & \multirow{2}{*}{$\begin{array}{c}\mathrm{H} 2 \\
-\end{array}$} & \multirow{2}{*}{$\begin{array}{ll}\mathrm{H} 3 \\
-\end{array}$} & \multirow{2}{*}{$\begin{array}{ll}\text { EO } \\
59\end{array}$} & \multirow{2}{*}{$\begin{array}{ll}\mathrm{ES} \\
32\end{array}$} & \multirow{2}{*}{$\begin{array}{ll}\mathrm{HC} \\
66\end{array}$} & \multirow{2}{*}{$\frac{P B}{141}$} & \multirow{2}{*}{$\begin{array}{ll}\mathrm{SH} \\
116\end{array}$} & \multirow{2}{*}{$\begin{array}{c}\text { SR } \\
172\end{array}$} & \multirow{2}{*}{\begin{tabular}{l|} 
Total \\
586
\end{tabular}} \\
\hline $\bar{n}$ & $\mathrm{H} 1$ & & & & & & & & & \\
\hline 恋 & $\mathrm{H} 2$ & - & - & 35 & 34 & 11 & 59 & 63 & 68 & 270 \\
\hline$\frac{0}{2}$ & EO & 28 & 78 & 5 & 8 & 2 & 22 & 38 & 14 & 195 \\
\hline 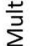 & ES & 32 & 28 & 6 & 9 & 1 & 9 & 11 & 11 & 107 \\
\hline in & $\mathrm{HC}$ & 13 & 30 & 9 & 2 & 3 & 9 & 33 & 6 & 105 \\
\hline 焉 & PB & 53 & 112 & 33 & 7 & 7 & 64 & 69 & 25 & 370 \\
\hline. & $\mathrm{SH}$ & 82 & 198 & 23 & 8 & 6 & 40 & 99 & 32 & 488 \\
\hline ð & SR & 62 & 140 & 25 & 7 & 9 & 26 & 59 & 20 & 348 \\
\hline \multicolumn{2}{|c|}{ Total } & 270 & 586 & 195 & 107 & 105 & 370 & 488 & 348 & 2469 \\
\hline & & & \multicolumn{2}{|c|}{$\begin{array}{l}\mathrm{H} 1 \text { - home in the beginning } \\
\mathrm{H} 2 \text { - home in the middle } \\
\mathrm{H} 3 \text { - home in the end }\end{array}$} & & \multicolumn{2}{|l|}{$\begin{array}{l}\text { EO - eat out } \\
\text { ES - escort } \\
\text { HC - health care }\end{array}$} & \multicolumn{2}{|c|}{$\begin{array}{l}\text { PB - person business } \\
\text { SH - shopping } \\
\text { SR - social recreation }\end{array}$} & \\
\hline
\end{tabular}

Now we convert the individual-episode file to an individual-episode-period file, which is illustrated in Figure 1. Initially, the duration times of 2,469 activity episodes of 586 retirees were grouped into 5-min intervals, yielding 81,828 observations in the 
converted data set, which caused a prohibitive computational burden. Therefore, we decided to reduce the file size by increasing the width of the time interval to 15 minutes, which produced 28,074 observations in the individual-episode-period file. Note that broadening the time interval does not change the number of episodes. If there are several episodes within a 15-min interval, all the episodes are retained in the 15-minute-interval data set as a duration time of one 15-min interval is recorded for each of them. The top panel of Figure 1 illustrates an individual who experienced three events of terminating a current activity episode and moving to a next activity episode for a day: from $\mathrm{H} 1$ to SR, from SR to $\mathrm{SH}$, and from $\mathrm{SH}$ to $\mathrm{H} 3$. The duration of the first activity H1 was 420 minutes, which accounts for 28 intervals in 15 -min time slots. This can be said that the first event of ending $\mathrm{H} 1$ and transitioning to SR occurred during the 28th 15-min time interval. As shown in the bottom panel of Figure 1, the one record of the event occurrence $\left(\mathrm{r}_{i j}=\mathrm{SR}\right)$ with the duration $\left(\mathrm{y}_{i j}=28\right)$ is converted to a sequence of 28 multinomial responses $\left(\mathrm{y}_{1 i j}, \mathrm{y}_{2 i j}, \ldots, \mathrm{y}_{27 i j}, \mathrm{y}_{28 i j}\right)=(0,0, \ldots, 0, \mathrm{SR})$. Similarly, the second activity episode ended in the 5 th time interval and is transformed with a series of 5 multinomial responses, while as the third episode terminated during the 1st interval, a single multinomial response is only necessary. In the converted data set, the response of zero represents no event occurrence during that time interval, which will be used as the reference category for the multinomial logit model. 
>> original data in an "individual-episode" format

\begin{tabular}{|c|c|c|c|c|}
\hline $\begin{array}{l}\text { individual } \\
\text { (j) }\end{array}$ & $\begin{array}{c}\text { episode } \\
\text { (j) }\end{array}$ & $\begin{array}{c}\text { state } \\
\text { (sij) }\end{array}$ & $\begin{array}{c}\text { event } \\
\text { (rij) }\end{array}$ & $\begin{array}{c}\text { duration } \\
\text { (yi) }\end{array}$ \\
\hline 80000791 & 1 & $\mathrm{H} 1$ & SR & 28 \\
\hline 80000791 & 2 & SR & SH & 5 \\
\hline 80000791 & 3 & $\mathrm{SH}$ & $\mathrm{H} 3$ & 1 \\
\hline \multicolumn{5}{|c|}{$\gg \gg$ converted data in an "individual-epidose-period" format } \\
\hline $\begin{array}{l}\text { individual } \\
\text { (j) }\end{array}$ & $\begin{array}{l}\text { episode } \\
\text { (i) }\end{array}$ & $\begin{array}{r}\text { state } \\
\text { (stij) }\end{array}$ & $\begin{array}{c}\text { event } \\
\text { (ytij) }\end{array}$ & $\begin{array}{l}\text { time interval } \\
\text { (t) }\end{array}$ \\
\hline 80000791 & 1 & $\mathrm{H} 1$ & 0 & 1 \\
\hline 80000791 & 1 & $\mathrm{H} 1$ & 0 & 2 \\
\hline 80000791 & 1 & $\mathrm{H} 1$ & 0 & 3 \\
\hline 80000791 & 1 & $\mathrm{H} 1$ & 0 & 4 \\
\hline 80000791 & 1 & $\mathrm{H} 1$ & 0 & 5 \\
\hline 80000791 & 1 & $\mathrm{H} 1$ & 0 & 6 \\
\hline 80000791 & 1 & $\mathrm{H} 1$ & 0 & 7 \\
\hline 80000791 & 1 & $\mathrm{H} 1$ & 0 & 8 \\
\hline 80000791 & 1 & $\mathrm{H} 1$ & 0 & 9 \\
\hline 80000791 & 1 & $\mathrm{H} 1$ & 0 & 10 \\
\hline 80000791 & 1 & $\mathrm{H} 1$ & 0 & 11 \\
\hline 80000791 & 1 & $\mathrm{H} 1$ & 0 & 12 \\
\hline 80000791 & 1 & $\mathrm{H} 1$ & 0 & 13 \\
\hline 80000791 & 1 & $\mathrm{H} 1$ & 0 & 14 \\
\hline 80000791 & 1 & $\mathrm{H} 1$ & 0 & 15 \\
\hline 80000791 & 1 & $\mathrm{H} 1$ & 0 & 16 \\
\hline 80000791 & 1 & $\mathrm{H} 1$ & 0 & 17 \\
\hline 80000791 & 1 & $\mathrm{H} 1$ & 0 & 18 \\
\hline 80000791 & 1 & $\mathrm{H} 1$ & 0 & 19 \\
\hline 80000791 & 1 & $\mathrm{H} 1$ & 0 & 20 \\
\hline 80000791 & 1 & $\mathrm{H} 1$ & 0 & 21 \\
\hline 80000791 & 1 & $\mathrm{H} 1$ & 0 & 22 \\
\hline 80000791 & 1 & $\mathrm{H} 1$ & 0 & 23 \\
\hline 80000791 & 1 & $\mathrm{H} 1$ & 0 & 24 \\
\hline 80000791 & 1 & $\mathrm{H} 1$ & 0 & 25 \\
\hline 80000791 & 1 & $\mathrm{H} 1$ & 0 & 26 \\
\hline 80000791 & 1 & $\mathrm{H} 1$ & 0 & 27 \\
\hline 80000791 & 1 & $\mathrm{H} 1$ & SR & 28 \\
\hline 80000791 & 2 & SR & 0 & 1 \\
\hline 80000791 & 2 & SR & 0 & 2 \\
\hline 80000791 & 2 & SR & 0 & 3 \\
\hline 80000791 & 2 & SR & 0 & 4 \\
\hline 80000791 & 2 & SR & $\mathrm{SH}$ & 5 \\
\hline 80000791 & 3 & $\mathrm{SH}$ & $\mathrm{H3}$ & 1 \\
\hline
\end{tabular}

Figure 3-1 Converting from Individual-Episode to Individual-Episode-Period 


\subsubsection{Model Development}

For the discrete-time duration variable or $y_{t i j}$, we develop a two-level multinomial logit model using Equation 3, which is re-written here:

$$
\begin{aligned}
& \log \left(\frac{h_{t i j}^{(r)}}{h_{t i j}^{(0)}}\right)=\alpha_{0}^{(r)}+\alpha_{1}^{(r)} \log (t)+\beta^{(r)} \text { State }_{t i j}^{(r)}+\gamma^{(r)} T O D_{t i j}+u_{j}^{(r)}, \\
& r \in\{H 2, H 3, E O, E S, H C, P B, S H, S R\}
\end{aligned}
$$

The first two terms of the right hand side of Equation 4 form the baseline logit-hazard. Instead of using temporal dummies, we use the parametric specification to make the model more parsimonious. Two parametric forms for the baseline logit-hazard were compared - quadratic and log, and it turned out that the model fit with the log form was better in this study. Both the overall intercept (i.e., $\alpha_{0}$ ) and the duration effect (i.e., $\left.\alpha_{1}\right)$ are specified to vary by event type $r$.

Dummy variables for origin activity are included to take into account multiple states from which an event occurs. Since some transitions are not allowed, different dummy coding schemes are used for each origin activity state. For the in-home destination types, five dummies (i.e., EO, ES, PB, SH, and SR) are defined for the origin state with $\mathrm{HC}$ as the reference category. For the out-of-home destinations, we create even origin state dummies (i.e., H2, EO, ES, HC, PB, SH, and SR), taking $\mathrm{H} 1$ as the reference case. As the coefficient of each origin state dummy is allowed to vary across event type $r$, it is expected to capture all the chaining effects from an origin from a destination. 
Time-of-day effects on the risk of an event are an important factor. Transitions to a particular type of new activity strongly depend on hours of day. For example, leaving a present activity for eating out usually occurs between 11:00 AM and 1:00 PM for lunch and between 5:00 PM and 7:00 PM for dinner. People tend to participate in social activities at certain times of day, say, in the evening. An easy way is to include dummy variables indicating hours of day. However, given that many state dummies are already specified in the model, adding a larger number of time-of-day dummies is not a good choice. Also, since the time-of-day dummies may be severely correlated, especially for adjacent hours of day, including them as predictors may lead to an unstable model. Alternatively, hours of day can be treated as a circular or periodic variable. To obtain a periodic variable of hour of day measured in degrees, hour of day $(\mathrm{H})$ is multiplied by $2 \pi / P$, where $P$ represents the known period of the periodic phenomenon (in our case 24 hours). The sine and cosine of the periodic variable are then inserted as explanatory variables. Therefore, the time-of-day effects are included as trigonometric predictors as follows:

$$
\gamma^{(r)} \operatorname{TOD}_{t i j}^{(r)}=\gamma_{1}^{(r)} \cos \left(\frac{2 \pi}{P} H\right)^{(r)}+\gamma_{2}^{(r)} \sin \left(\frac{2 \pi}{P} H\right)^{(r)}
$$

The coefficients $\gamma_{1}$ and $\gamma_{2}$ may vary across competing risk $r$. Higher order trigonometric polynomials of the periodic variable can be explored as possible independent variables for each event type $r$. 
Lastly, $u_{j}^{(r)}$ is the upper-level random effect for the contrast of response category $\mathrm{r}$ with the reference category 0 . We add eight random effects; one for each destination type, and it is assumed that the random effects are multivariate normally distributed with a vector of zero means and a variance-covariance matrix. Non-zero variances suggest that there exist unobserved individual-level influences on the odds of being in a response category $r$ rather than the reference category 0 , while non-zero covariances capture correlation between the unobserved individual-specific influences of the different response categories.

\subsection{Results}

This section describes the model estimation results. It is important to note that our model specification described in the previous section is not a "final" one. Since the aim of this paper is to demonstrate the potential of a discrete-time approach to modeling activity duration, we simplify the model structure by including only a few fixed effects (duration, state, and time-of-day effects) together with random effects. As shown in Table 3, the log-likelihood statistics were acceptable, particularly for a model with a large number of choice alternatives. 
Table 3-3 Model Fit Statistics

\begin{tabular}{lc}
\hline \multicolumn{1}{c}{ Statistic } & \multicolumn{1}{c}{ Value } \\
\hline Log likelihood at null & -61684.88 \\
Log likelihood at constants & -11858.07 \\
Log likelihood at two-level convergence & -10556.70 \\
Rho Squared w.r.t null & 0.829 \\
Rho Squared w.r.t constants & 0.110 \\
Number of cases & 28,074 \\
\hline
\end{tabular}

Note: w.r.t $=$ with regard to

\subsubsection{Random Effects}

For comparison we fitted a single-level multinomial logit model (which is not shown in this paper) that ignores possible correlations between activity episodes participated by an individual for a day. Overall, we found little differences in parameter estimates between single-level and multi-level models, even if the standard errors in the single-level model were mostly underestimated as expected. We also conducted a likelihood ratio test to see if the multilevel model performed statistically better than the single-level model. The chisquare statistic of 140.67 with 36 degrees of freedom indicated as a whole the significance of the random-effect parameters, $\operatorname{var}\left(u^{r}\right)$ and $\operatorname{cov}\left(u^{r}, u^{q}\right)$ for $r, q \in\{\mathrm{H} 2, \mathrm{H} 3$, $\mathrm{EO}, \mathrm{ES}, \mathrm{HC}, \mathrm{PB}, \mathrm{SH}, \mathrm{SR}\}$ and $r \neq q$.

Table 4 shows the variance-covariance matrix of the estimated random effects. There was between-individual variation in the choice of returning home temporarily $(\mathrm{H} 2)$ versus staying (ST), returning home in the end (H3) versus staying (ST), and escorting (ES) versus staying (ST), as indicated by significant non-zero variances. However, there was no significant evidence of unobserved individual-specific influences on the hazard of 
the other out-of-home activity purposes (EO, HC, PB, SH, and SR) versus staying (ST). In addition, we found that there is a significant correlation between the random effects for some pairs of transitions. During a day, for example, individuals with a high hazard of moving from staying (ST) to health care $(\mathrm{HC})$ tend to have a high hazard of moving from staying (ST) to eat-out (EO), as indicated by its positively significant covariance $(0.89)$. For many other pairs of transitions, however, the correlations between the random effects were not significant.

Table 3-4 Random Effects Variance-Covariance Matrix

\begin{tabular}{l|llllllll}
\hline & ST to H2 & ST to H3 & ST to ES & ST to EO & ST to HC & ST to PB & ST to SH & ST to SR \\
\hline ST to H2 & $0.66^{* * *}$ & & & & & & & \\
ST to H3 & 0.09 & $1.69 * * *$ & & & & & & \\
ST to ES & 0.26 & $0.49 * * *$ & $0.51 * * *$ & & & & & \\
ST to EO & 0.12 & -0.13 & 0.06 & 0.08 & & & & \\
ST to HC & -0.17 & 0.33 & -0.22 & $0.89 *$ & -0.28 & & & \\
ST to PB & -0.16 & -0.19 & $0.43 * *$ & -0.12 & 0.49 & -0.40 & & \\
ST to SH & 0.09 & $-0.30 * *$ & 0.26 & 0.30 & -0.04 & 0.02 & 0.05 & \\
ST to SR & $0.50 * *$ & -0.18 & -0.04 & -0.22 & -0.11 & 0.11 & 0.02 & -0.05 \\
\hline
\end{tabular}

\subsubsection{Fixed Effects}

Table 5 shows the estimated coefficients and standard errors for the fixed part of the full model. The results indicate that the duration effects were significantly positive for all event types of destination activity after controlling for the origin activity states and the times of day. This is as expected because the risk of terminating a current activity and then moving to a next activity increases as the time spent in the current activity episode increases regardless of activity types. For example, the odds of returning to home temporarily $(\mathrm{H} 2)$ versus staying in a current activity increased by $e^{0.31}=1.36$ with 
respect to a natural $\log$ of one 15 -min time interval increment in a current activity episode.

Most of the state dummy variables were statistically significant for most destination activity types. For example, we found significant positive transition relationships for returning to home temporarily from escort (3.99), personal business (1.39) and shopping (1.54), not from eat-out (0.65) and social recreation (0.11). On further examination of the results, we found that the odds of moving to eat out rather than staying was $e^{2.99}=19.89$ times higher when people are at home in the middle of day $(\mathrm{H} 2)$ than in the beginning of day $(\mathrm{H} 1)$. This implies that retirees tend to perform more than one tour for a day and during the second or third tour they are more likely to eat out for lunch or dinner. 
Table 3-5 Estimated Coefficients

\begin{tabular}{|c|c|c|c|c|c|c|c|c|c|c|c|c|c|c|c|c|}
\hline \multirow[b]{3}{*}{ Variable } & \multicolumn{16}{|c|}{ Destination Activity Type } \\
\hline & \multicolumn{2}{|c|}{$\begin{array}{c}\text { H2 } \\
\text { (vs. Stay) }\end{array}$} & \multicolumn{2}{|c|}{$\begin{array}{c}\text { H3 } \\
\text { (vs. Stay) }\end{array}$} & \multicolumn{2}{|c|}{$\begin{array}{c}\text { EO } \\
\text { (vs. Stay) }\end{array}$} & \multicolumn{2}{|c|}{$\begin{array}{c}\text { ES } \\
\text { (vs. Stay) }\end{array}$} & \multicolumn{2}{|c|}{$\begin{array}{c}\text { HC } \\
\text { (vs. Stay) }\end{array}$} & \multicolumn{2}{|c|}{$\begin{array}{c}\text { PB } \\
\text { (vs. Stay) }\end{array}$} & \multicolumn{2}{|c|}{$\begin{array}{c}\text { SH } \\
\text { (vs. Stay) }\end{array}$} & \multicolumn{2}{|c|}{$\begin{array}{c}\text { SR } \\
\text { (vs. Stay) }\end{array}$} \\
\hline & Est. & S.E. Sig. & Est. & S.E. Sig. & Est. & S.E. Sig. & Est. & S.E. Sig. & Est. & S.E. Sig. & Est. & S.E. Sig. & Est. & S.E. Sig. & Est. & S.E. Sig. \\
\hline $\begin{array}{l}\text { Constant } \\
\text { Duration Effect }\end{array}$ & -4.55 & $0.39 * * *$ & -2.49 & $0.26 * * *$ & -8.46 & $0.46^{* * *}$ & -10.32 & $0.75 * * *$ & -12.37 & $0.99 * * *$ & -7.90 & $0.40 * * *$ & -7.46 & $0.33 * * *$ & -7.67 & $0.39 * * *$ \\
\hline $\begin{array}{l}\log (\text { actDur15) } \\
\text { State Effect }\end{array}$ & 0.31 & $0.10 * *$ & 0.18 & $0.07 * *$ & 0.84 & $0.11 * * *$ & 0.97 & $0.16 * * *$ & 1.60 & $0.25 * * *$ & 0.68 & $0.10 * * *$ & 0.48 & $0.08 * * *$ & 1.04 & $0.10 * * *$ \\
\hline this $\mathrm{H} 2$ & & & & & 2.99 & $0.33 * * *$ & 3.08 & $0.42 * * *$ & 3.32 & $0.59 * * *$ & 2.67 & $0.26 * * *$ & 2.64 & $0.24 * * *$ & 3.21 & $0.28 * * *$ \\
\hline thisHC & & & & & 3.73 & $0.46 * * *$ & 2.54 & $0.93 * *$ & 2.57 & $0.93 * *$ & 2.73 & $0.43 * * *$ & 3.44 & $0.31 * * *$ & 2.89 & $0.52 * * *$ \\
\hline thisEO & 0.65 & 0.37 & 0.20 & 0.26 & 2.39 & $0.53 * * *$ & 3.85 & $0.56 * * *$ & 3.39 & $0.98 * * *$ & 3.29 & $0.36 * * *$ & 3.50 & $0.30 * * *$ & 2.88 & $0.38 * * *$ \\
\hline thisES & 3.99 & $0.44 * * *$ & 2.93 & $0.38 * * *$ & 7.21 & $0.61 * * *$ & 6.75 & $0.72 * * *$ & 7.45 & $1.43 * * *$ & 6.70 & $0.59 * * *$ & 6.66 & $0.51 * * *$ & 7.57 & $0.56 * * *$ \\
\hline thisPB & 1.39 & $0.34 * * *$ & 0.83 & $0.27 * *$ & 5.04 & $0.43 * * *$ & 3.98 & $0.66 * * *$ & 5.04 & $0.97 * * *$ & 3.92 & $0.36 * * *$ & 4.23 & $0.30 * * *$ & 4.21 & $0.41 * * *$ \\
\hline thisSH & 1.54 & $0.33 * * *$ & 0.94 & $0.23 * * *$ & 4.23 & $0.40 * * *$ & 4.35 & $0.65 * * *$ & 4.52 & $0.78 * * *$ & 3.55 & $0.35 * * *$ & 3.99 & $0.26 * * *$ & 4.19 & $0.37^{* * *}$ \\
\hline $\begin{array}{l}\text { thisSR } \\
\text { Time-of-day Effec }\end{array}$ & 0.11 & 0.33 & -0.50 & $0.26 *$ & 2.47 & $0.32 * * *$ & 2.04 & $0.53 * * *$ & 3.06 & $0.60 * * *$ & 1.96 & $0.33 * * *$ & 2.55 & $0.23 * * *$ & 1.45 & $0.33 * * *$ \\
\hline hSIN1 & 1.43 & $0.13^{* * *}$ & -0.01 & 0.11 & 0.92 & $0.17 * * *$ & 1.15 & $0.21 * * *$ & 2.31 & $0.29 * * *$ & 0.87 & $0.13 * * *$ & 0.60 & $0.10 * * *$ & 1.56 & $0.12 * * *$ \\
\hline hcos1 & -0.66 & $0.29 *$ & 0.31 & $0.12 * *$ & 0.01 & 0.24 & 0.51 & $0.26 *$ & -1.22 & 0.65 & -0.83 & $0.21 * * *$ & -1.08 & $0.18 * * *$ & 0.70 & $0.17^{* * *}$ \\
\hline
\end{tabular}




\subsection{Conclusion and Future Work}

In this paper we explicitly proposed a hazard-based duration model in a discrete-time framework for activity duration analysis that incorporated time dependencies into activity episode choice and duration. We also demonstrated the fact that the discrete-time hazardbased duration model is essentially equivalent to a discrete choice model with temporal dummies as the simplest form of dynamic discrete choice models. In addition, we illustrated the flexibility of the discrete-time approach by enabling complex situations of activity-travel data to be modeled, such as multiple states of origin activity, competing risks of destination activity, and a multilevel structure for recurrent activity episodes of an individuals and by facilitating the integration of activity participation model with supply model of dynamic traffic assignment (DTA).

Recently, the integration between activity-based demand models and dynamic network supply models becomes a key issue as travel modeling research and practice adopt the microsimulation framework. Two approaches are available for the demandsupply integration: sequential integration and dynamic integration (Konduri, 2012). In the traditional sequential integration approach, the demand and supply components are run independently and sequentially in the form of an input-output data flow with a feedback of the network conditions until convergence is achieved. The dynamic integration approach adopts an "event-based paradigm" while constantly communicating between the demand and supply models along a continuous time axis. In other words, the demand model sends a set of departing trips to the supply model at every simulation minute for those travelers who decide to move to a next activity location. The DTA supply model 
loads these trips on the network. For those travelers who arrive at their destination, the supply model feeds back a set of arriving trips to the demand model in every minute of simulation. Then the demand model simulates a series of activity participation decisions, including activity duration. As the state of practice for activity duration modeling is the continuous-time method, we see a strong potential of the proposed discrete-time method that can make the demand model to be dynamic or time-dependent as well. As such, in a dynamic integration, the dynamic network model can be integrated with the dynamic demand model with the discrete-time framework, resulting in "truly emergent" activitytravel participation decisions.

There is a list of works we would like to pursue in our future research. First, as mentioned previously, one important advantage of the discrete-time approach over the continuous-time approach is the capacity to explicitly include time-varying independent variables. To make the model more realistic, we hope to include more policy-sensitive time-varying covariates in our model specification. For example, one can trace the availability of household auto(s), the presence of household kid(s) at home or school, and the jointness of other household member(s) into discrete-time intervals. Time-varying accessibility measures, proposed in recent ABM model development, can be included as another set of covariates to explicitly capture the temporal variation of activity participation opportunities (Paleti et al., 2015). Second, we added the upper-level random effects to control for unobserved individual-specific influences as well as correlation between the unobserved individual-specific influences. Possible correlation between the random effects was rarely detected for our population group (i.e., retirees). However, it is 
expected to observe high correlation in random effects for other population groups, such as workers and students. Alternative model specification with assumed correlation structure beforehand may be better at capturing the correlation structure among these population groups. Lastly, a potential problem with the discrete-time approach is that data in the person-episode-period format can be extremely large, particularly when time intervals are short and the observation period is long. One strategy to reduce the data size is to group time intervals and then weight the grouped observations by exposure time (Steele, Goldstein, \& Browne, 2004). 


\section{MULTIPLE IMPUTATION BY CHAINED EQUATIONS}

As analysis and models for land use and transportation planning gradually move to micro-level because of policy requirements and theoretical and technical advantages (Waddell, 2009), missing data becomes an increasingly common problem in micro-level land use and transportation modeling and policy analysis. According to the study of Waddell et al. (2004), up to $70 \%$ of the efforts in land use and transportation modeling are spent on data processing, and handling missing data is a major piece of these efforts.

Missing values in land use and transportation data sets, particularly those in land

use data sets, are generally handled on an ad-hoc basis with either manual inspection and cleaning, or rule-based or heuristic methods. While these methods can solve one type of missing data problem at a time in some user-specified sequence, they are hard to be adapted for different applications and there is no systematic way to assess their quality (Waddell, 2009).

More sophisticated statistical modeling and machine learning techniques have been developed in statistics and computer sciences and tested and applied to tackle missing data problems in many fields such as industrial engineering (Lakshminarayan et al., 1999), forestry (Eskelson et al., 2009), etc. Waddell (2009) suggests that k-nearest neighbors and support vector machine may be two promising techniques for imputing missing land use data, particularly, parcel-level data. However, to our knowledge, there is no systematic research assessing the applicability and quality of these data imputation techniques in imputing missing land use data. 
Furthermore, most applications of data imputation in land use data generate a single imputation of the missing value - that is, substitute a missing value with a single best guess. However, single imputation masks the uncertainty in the missing values. In other words, the imputed values in the data are treated as if they are real observed values, without appropriately addressing the uncertainty associated with the substitutions. To this end, Rubin (Rubin, 1987) proposes a multiple imputation framework. Instead of producing a single best guess, multiple imputation uses a Monte Carlo simulation and replaces each missing entry with a set of $m$ plausible values in which $m$ is typically small, say 3 to 10 . The advantage of multiple imputation is that, each of the $m$ imputed data sets can still be analyzed with standard complete-data methods, but the analysis results can be combined by simple arithmetic calculations to produce mean estimates, standard errors, and p-value for quantities of interest that incorporate uncertainty due to imputation of missing data.

A popular approach to implementing multiple imputation, especially for multivariate missing data, is multiple imputation by chained equations (MICE), also known as fully conditional specification or sequential regression multiple imputation. It has been widely applied in psychology (Azur et al., 2011), medicine (White et al., 2010), epidemiology (Burgette \& Reiter, 2010), etc. One of the reasons for the popularity of MICE is its flexibility. MICE can handle missing continuous and discrete variables because each variable with missing data is imputed based on its own imputation model. Recent studies attempt to implement recursive partitioning within MICE in order to 
automatically preserve interaction effects in the data (Doove et al., 2014; Shah et al., 2014).

In this study, we apply the non-parametric MICE approach to impute missing values in parcel data. Recently more and more land use and transportation modeling work and analysis moves to use parcel data (Waddell, 2009). However, parcel data are prone to problems of incomplete or missing values. In addition, in land use and transportation modeling, base year data are usually used to project future land use and travel patterns. So even though the number of missing values may be small, it is still necessary to impute them. We aim to test different methods in imputing missing parcel data and assess the quality of the imputation results, taking the uncertainty into consideration.

The remaining of this paper is organized as follows. The next section overviews the background of missing data patterns and mechanisms, multiple imputation, MICE, and the implementation of recursive partitioning in MICE. In the Parcel Data Imputation section, we discuss multiple imputation for parcel data, along with missing data description, MICE set-up, and visual diagnosis. The section that follows, we discuss the validation of non-parametric MICE. Finally, we conclude the paper with some suggestions for future research.

\subsection{Background}

\subsubsection{Patterns and Mechanisms of Missing Data}

For practical reasons, it is useful to distinguish three different patterns of missing data: univariate, monotone, and arbitrary patterns (Schafer \& Graham, 2002; Van Buuren, 
2007). First, a missing data pattern is univariate if only one variable has missing values and the remaining variables are all completely observed. For the univariate missing data, a variety of parametric or non-parametric methods can be used for conducting multiple imputation. Second, in the monotone pattern, more than one variable are missing and the missing variables can be ordered such that if a variable is missing for a subject, then other variables are also missing for that subject. Such a monotone pattern is often observed in a longitudinal data set as one leaves the longitudinal study in the middle of entire waves of data collection. In this case, it is possible to impute the multivariate missing data by a series of univariate methods for multiple imputation. Lastly, an arbitrary pattern is observed from multivariate missing variables, in which their missing values can occur in any set of variables for any subjects. Then, we need a truly multivariate method for multiple imputation. The arbitrary pattern is the focus of this research.

On the other hand, it is important to understand different mechanisms through which data are missing, because different missing mechanisms may cause different risks of bias when missing data are excluded in analysis. The missing mechanisms are commonly classified as missing completely at random (MCAR), missing at random (MAR), and missing not at random (MNAR). First, MCAR assumes that the probability of data being missing depends on neither observed nor unobserved variables. In this case, the set of subjects that are completely observed is also a random sample from the source population. Thus, a complete case analysis in which all missing data are excluded gives unbiased results, even if such a simple method is less efficient because the entire data set is not used. Second, in the MAR mechanism, the probability that data are missing 
depends on observed variables, but not on unobserved ones. In this case, a complete case analysis is no longer based on a random sample, and selection biases are likely to occur. Such a bias can be overcome through data imputation, in which a missing value for a subject is replaced by a predicted value from the subject's other known characteristics. Lastly, a mechanism for missing data is MNAR if the probability of data being missing depends on both observed and unobserved variables. In this case, there is no universal method. We may only address the MNAR biases by conducting sensitivity analyses in which the effects of different mechanisms are compared (Donders et al., 2006). As in many other studies, the MAR mechanism is assumed in this study to apply a data imputation approach to the missing data problems.

\subsubsection{Multiple Imputation}

Multiple Imputation is a statistical approach to handling missing data, which was first proposed in the 1980s by Rubin (1987). It aims to overcome the limitation of single imputation by allowing for the uncertainty introduced by missing data. Multiple imputation function is increasingly available in common statistical software, such as R, SAS, Stata, and SPSS. In general, multiple imputation consists of three steps. The first step is to construct an imputation model, which is sometimes referred to as an imputer or an imputation engine. For a single missing variable $z$, the imputer regresses $z$ on a set of non-missing variables among individuals with observed $z$ values. Now each missing value of $\mathrm{z}$ is replaced $m$ times by a plausible value that is a random draw from the predictive distribution of the imputation model. 
The second step is usually simple. Each of the multiple imputed data sets is analyzed separately but identically by a complete-data method (e.g., a linear regression) to estimate quantities of interest (e.g., the regression coefficients). Since the multiple data sets are identical for the complete data but not for the imputed data, this step generates the $m$ different estimates for each quantity.

The third step is to combine the $m$ estimates, using Rubin's rules (Rubin, 1987). Suppose that $\hat{\theta}_{j}$ is an estimate of a scalar quantity of interest obtained from imputed data set $j(j=1,2, \ldots, m)$ and $W_{j}$ is the variance of $\hat{\theta}_{j}$. The overall estimate $\hat{\theta}$ is simply the average of the individual $m$ estimates:

$$
\hat{\theta}=\frac{1}{m} \sum_{j=1}^{m} \hat{\theta}_{j}
$$

The overall variance of the overall estimate $\hat{\theta}$ is formulated with two components: the within-imputation variance (W) and the between-imputation variance (B):

$$
\operatorname{var}(\hat{\theta})=W+\left(1+\frac{1}{m}\right) B
$$

The within-imputation variance explains the variation of the estimate in one imputed data set, which is calculated by averaging the $m$ variances: 


$$
W=\frac{1}{m} \sum_{j=1}^{m} W_{j}
$$

The between-imputation variance measure how the estimates vary from imputation to imputation to reflect the uncertainty due to missing data, which can be given:

$$
B=\frac{1}{m-1} \sum_{j=1}^{m}\left(\hat{\theta}_{j}-\hat{\theta}\right)^{2}
$$

The greater the variation of the estimates across the imputations, the higher the uncertainty introduced by missing data. The overall standard error is the square root of

$\operatorname{var}(\hat{\theta})$. A significance test of the null hypothesis $\theta=0$ is performed in a usual way. Since single imputation omits the between-imputation variance, the standard error is always too small (White et al., 2010).

\subsubsection{Multiple Imputation by Chained Equations (MICE)}

In large data sets with missing values such as the parcel data set, the missing values often occur in more than one variable and follow an arbitrary pattern. When the pattern of the multivariate missing data is arbitrary, two general multivariate approaches are available for multiple imputation: joint modeling (JM) and multiple imputation by chained equations (MICE). The JM approach assumes a multivariate normal distribution for all variables in the imputation model. Imputed values are obtained from the estimated joint 
distribution. JM was first proposed by Schafer (Schafer, 1997) and was widely used in the earlier applications of multiple imputation to multivariate missing data. However, the parametric method of JM may lack flexibility due to its normality assumption. In case that there are both continuous and discrete variables, the assumption of multivariate normality is often violated. The MICE approach is more flexible in that, instead of assuming multivariate normality, MICE models a series of conditional distributions, one for each missing variable, based on the other variables in the imputation model. The semi-parametric method of MICE is increasing popular and there are many statistical packages available for MICE (Van Buuren, 2007).

To describe the procedure of generating multiple imputed data sets in MICE, suppose a set of variables, $y_{l}, \ldots, y_{j}$, where some of them are missing. The following steps are involved (Azur et al., 2011):

Step 1: Fill in every missing value by a random draw from the observed values, which will serve as a placeholder.

Step 2: For the first missing variable, say $y_{l}$, return the placeholders to missing, and then construct an imputation model that regresses $y_{l}$ on the other variables, say $y_{2}, \ldots, y_{j}$, only among individuals with the observed $y_{1}$.

Step 3: Replace every missing value in $y_{l}$ by a random draw from the posterior predictive distribution of the imputation model for $y_{1}$.

Step 4: For the second missing variable, say $y_{2}$, return the placeholders to missing, and then construct an imputation model that regresses $y_{2}$ on the previously 
imputed variable(s) $y_{1}$ and the other variables $y_{3}, \ldots y_{j}$, only among individuals with the observed $y_{2}$.

Step 5: Replace every missing value in $y_{2}$ by a random draw from the posterior predictive distribution of the imputation model for $y_{2}$.

Step 6: For all other missing variables, repeat Steps 4 and 5.

Step 7: To stabilize the results, repeat Steps 2 through $6 l$ times (e.g., 10 to 20), which produces one imputed data set.

Step 8: Repeat Steps 1 through $7 m$ times (e.g., 5 to 10) to generate $m$ imputed data sets.

The most significant advantage of using MICE is its ability to handle different types of variables because each missing variable is imputed from its own imputation model. For example, a linear regression imputer can be used to impute missing values in a continuous variable, while a logistic regression imputation model may be constructed to impute a discrete missing variable. When a missing variable is skewed and its transformation to normality is impossible, Predictive Mean Matching (PMM) is usually suggested as an imputer. PMM can be seen as a type of random k-nearest-neighbor method (Waddell, 2009). In a PMM model, imputed values are simply sampled from the observed values of the missing variable, so that the distribution of the imputed values is similar to that of the observed values. Especially, PMM is desirable when the sample size is large and a missing variable is 'semi-continuous' for which many values are equal (White et al., 2010). 


\subsubsection{Recursive Partitioning in MICE}

In MICE, it is required to build an imputation model for each of the variables with missing values. In practice, however, specifying the imputation models is not an easy task for at least two reasons. First, missing variables to be imputed may have complex distributions. In this case, standard parametric models are not appropriate for the imputers. Also, data transformations to normality are not always possible. Second, there may exist interactive and nonlinear relationships among the variables in the imputers. The nature of these interactions and nonlinearity is usually unknown a priori. It is very laborious to add the interaction and nonlinear effects to the imputation models. To mitigate these two challenges, recent studies suggest the use of recursive partitioning as an imputation engine within MICE (Burgette \& Reiter, 2010; Doove, Van Buuren, \& Dusseldrop, 2014; Shah et al., 2014).

One of the most popular recursive partitioning techniques is Classification And Regression Trees (CART) (Breiman et al., 1984). CART is called either classification trees or regression trees, depending on the response variable of interest being categorical or continuous, respectively. In CART, a predictor space is partitioned so that observations are clustered into several groups that are relatively homogeneous with respect to the response variable. The best partitions are found by recursive binary splits of the predictors. The resulting series of splits are represented in a tree structure. The leaves of the tree represent the groups of observations, and the values in each leaf approximate the conditional distribution of the response variable based on the predictors. Since all splits are conditional on the previous splits, complex interactions are automatically 
detected in the tree. The grown tree is usually pruned to avoid overfitting and for easier interpretation. However, when a tree is built for imputation, this pruning procedure is not desirable (Burgette \& Reiter, 2010).

However, there are two major disadvantages of CART because of its hierarchical nature. First, the trees of CART may be suboptimal. A "best" split is determined only locally in each leaf regardless of future splits. Second, CART may produce unstable trees because trees may vary markedly from sample to sample. In other words, small changes in sample may lead to different initial splits and then yield quite different final trees. Alternatively, another popular recursive portioning method is Random Forests. While CART builds a single tree, Random Forests generates multiple trees and averages them (Breiman, 2001). Variation in the individual trees is produced by bootstrapping and random variable selection. Instead of using all observations, a bootstrap sample is used to build each tree. Rather than using all variables, a subset of variables is randomly selected to find the best split at each leaf (Doove et al., 2014).

\subsection{Case Study: Parcel Data Imputation}

Integrated land-use and transportation models are trending towards using disaggregated spatial resolution, such as parcel. Although some concerns for using micro-level data have been pointed out, such as large data requirements, long computing times, and stochastic variations (Wegener, 2011), the advantages of using parcels as the spatial unit of analysis are enormous. First, parcels are more behaviorally realistic in modeling the built environment than uniform grid cells as well as coarse zones. In the real world, 
transaction, regulation, and development usually occur at the parcel level. Second, using the parcel geography allows us to measure walking-scale accessibility by representing people's activity locations on parcels. Walking access to transit stops or grocery stores are increasingly important from public health and policy perspectives (Waddell, 2009).

In the U.S., parcel data are usually managed by local governments that assess property taxes. There is huge variation in data completeness and data quality across jurisdictions and missing values are common in variables that are necessary for developing integrated models, including building square feet, building type, number of stories, year built, building value, land value, land use, and so on. Especially for parcels that are exempt from property taxes but nevertheless important for models, there tend to be more missing values on the attributes. Therefore, there is a significant need for handling missing values in parcel data as the research and practice of integrated models move to use parcel as the spatial boundary.

For this study, we pick Multnomah County in Oregon as our study area. Multnomah County includes Oregon's largest city, Portland. The parcel data are obtained from Metro's Regional Land Information System (RLIS) that provides a variety of geographic information for the Portland metropolitan area. Metro and the regional partners update the parcel data every quarter. For this study we focus on one parcel data set updated in the fourth quarter of year 2011. The parcel data set provides information such as parcel area in square feet, building floor area in square feet, building value in dollar, land value in dollar, land use type, and so on. We detect a modest amount of missing entries from the parcel data set. The missing data pattern is shown in Table 1. 
Among the total of 271,977 parcels, $83.7 \%$ are completely observed for all of the variables. The remaining parcels have at least one missing entry for any of the variables. The largest number of missing entries (i.e., 37,037 or 13.6\%) is observed in the landvalue variable, while the smallest number of missing entries (i.e., 2,097 or $0.8 \%$ ) is in the land-use variable. Among the parcels with missing entries, $60.6 \%$ are missing for only one variable, $37.3 \%$ for two variables, and $2.2 \%$ for three variables. We also notice that the missing entries are scattered among the variables. Therefore, we conclude that the missing values in the parcel data set follows an arbitrary pattern, which makes MICE a suitable methods for imputing the multivariate missing values in the parcel data set.

Table 4-1 Missing Data Pattern of 271,977 Parcels $(1=$ observed \& 0 = missing $)$

\begin{tabular}{ccccccr}
\hline Parcel Area & Land Use & Building Value & Building Floor Area & Land Value & Frequency \\
\hline 1 & 1 & 1 & 1 & 1 & 227,591 \\
1 & 0 & 1 & 1 & 1 & 2,097 \\
1 & 1 & 1 & 0 & 1 & 2,825 \\
1 & 1 & 0 & 1 & 1 & 13 \\
1 & 1 & 1 & 1 & 0 & 21,959 \\
1 & 1 & 0 & 0 & 1 & 2,414 \\
1 & 1 & 1 & 0 & 0 & 14,122 \\
1 & 1 & 0 & 1 & 0 & 1 \\
& 1 & 1 & 0 & 0 & 0 & 955 \\
\hline Total & none & 2,097 & 3,383 & 20,316 & 37,037 & 271,977 \\
\hline
\end{tabular}

\subsubsection{Setting Up MICE: Choice of Predictors}

Assuming the MAR mechanism and arbitrary missing pattern, we now set up MICE to impute all the multivariate missing values on the parcels of Multnomah County. The first step is to determine which variables are included as predictors in the imputation models. Table 2 is a list of the variables. We include all of the four missing variables: building 
floor area, building value, land value, and land use. Since the first three continuous variables include huge values from which it is hard to draw meaningful interpretations, we scale down by dividing each of the three variables by parcel area. As a result, we use three new missing variables, namely, building floor area per parcel area (i.e., FAR; floor area ratio), building value per parcel area (i.e., BVPA), and land value per parcel area (i.e., LVPA). It is also important to note that these three missing variables are semicontinuous because a large portion of their values is zero and the remaining portion is continuous. The other missing variable (i.e., LU) is categorical with 9 levels, including agriculture, commercial, forest, industrial, multifamily residential, public/semi-public, rural, single family residential, and vacant. Additionally, four non-missing variables are included to increase the prediction power of the imputation models: Area, District, POP10, and JOB11. Area is a continuous predictor, representing the parcel size in square feet, while District is a categorical predictor with 10 polygons covering Multnomah County among 20 districts that divide the Portland Metropolitan area for a variety of planning and analysis purposes. The other two non-missing variables are obtained from outside data sources. POP10 represents the 2010 population size of census block group that the parcel belongs to, which is obtained from U.S. Census Data. JOB11 or number of jobs in census block group that the parcel belongs to is extracted from the 2011 Workplace Area Characteristics (WAC) file of Longitudinal Employer-Household Dynamics (LEHD). We use these two outside variables as a proxy for neighborhood characteristics of parcels. 
Table 4-2 A List of Predictors Included in the MICE Imputers

\begin{tabular}{|c|c|c|c|c|c|c|c|}
\hline $\begin{array}{c}\text { Variable } \\
\text { Name }\end{array}$ & Description & $\begin{array}{c}\text { Variable } \\
\text { Type }\end{array}$ & $\begin{array}{c}\text { Number of } \\
\text { Missing Parcels }\end{array}$ & Min & Max & Mean & Median \\
\hline FAR & building floor area per parcel area (in square feet) & semi-continuous & $20,316(7.5 \%)$ & 0 & 4,457 & 1.22 & 0.25 \\
\hline BVPA & building value per parcel area (in dollar) & semi-continuous & $3,383(1.2 \%)$ & 0 & 588,178 & 278.36 & 20.05 \\
\hline LVPA & land value per parcel area (in dollar) & semi-continuous & $37,037(13.6 \%)$ & 0 & 2,232 & 19.42 & 16.47 \\
\hline LU & 9 land use types & categorical & $2,097(0.8 \%)$ & - & - & - & - \\
\hline Area & parcel area (in square feet) & continuous & no missing & 2 & $30,956,896$ & $35,772.12$ & $5,433.68$ \\
\hline Disctrict & 10 districts covering Multnomah County & categorical & no missing & - & - & - & - \\
\hline POP10 & 2010 population size by Census block group & continuous & no missing & 8 & 4,746 & $1,657.36$ & $1,407.00$ \\
\hline JOB11 & 2011 number of jobs by Census block group & continuous & no missing & 4 & 30,390 & $1,173.45$ & 263.00 \\
\hline
\end{tabular}




\subsubsection{Setting Up MICE: Choice of Imputers}

The aim of this study is to impute missing values in four variables, namely, FAR, BVPA, LVPA, and LU using MICE. The key to success of a MICE application is how well the imputation model is specified for each missing variable. In the parcel data set described previously, missing values are observed in a mix of continuous and categorical variables with complex distributions. Especially, three of them (i.e., FAR, BVPA, and LVPA) are continuous but inflated with zeroes. In addition, there may exist a variety of interactions between the predictors. For example, we can easily think of the interaction effects of land use type and district geography on the building floor area ratio on a parcel. Multifamily residential parcels located in CBD district tend to have a higher FAR (i.e., a higher building on a smaller size of parcel) than the same land-use type of parcels in other districts. Manually testing such interaction effects in the imputation model is a very timeconsuming task with no guarantee of success. For these reasons, we initially consider two recursive partitioning methods (i.e., CART and Random Forests) as an imputation model of MICE. We generate 5 imputed data sets $(m=5$ in Step 8$)$ after 5 iterations $(l=5$ in Step 7).

\subsubsection{Visual Diagnosis}

Because we never observe the missing values, we cannot quantitatively assess how well each imputation technique performs. An important step in multiple imputation is to diagnose whether imputations are plausible or not. As a simple visual way of checking the plausibility of the imputations, it is useful to compare the distributions of original data 
and imputed data. Figure 1 shows the distributions of the three continuous variables as individual (jittered) points, in which blue points are observed data and red points are imputed data. The zeroth imputation of all the panels contains blue points only, representing the original distribution. The red points follow the blue points reasonably well without showing any data points that are clearly impossible. However, it should be noticed that the CART-based MICE did not produce proper imputes for extreme values of FAR and BVPA. They are severely right skewed compared with LVPA; the skewness of FAR, BVPA, and LVPA is 265, 92 and 21, respectively. For more graphical diagnostic tools, refer to Abayomi et al. (2008). To be able to quantitatively assess the performance of each imputation model, we will create a validation data set and compare how well each imputation recover the true values, which will be discussed in the next section. 


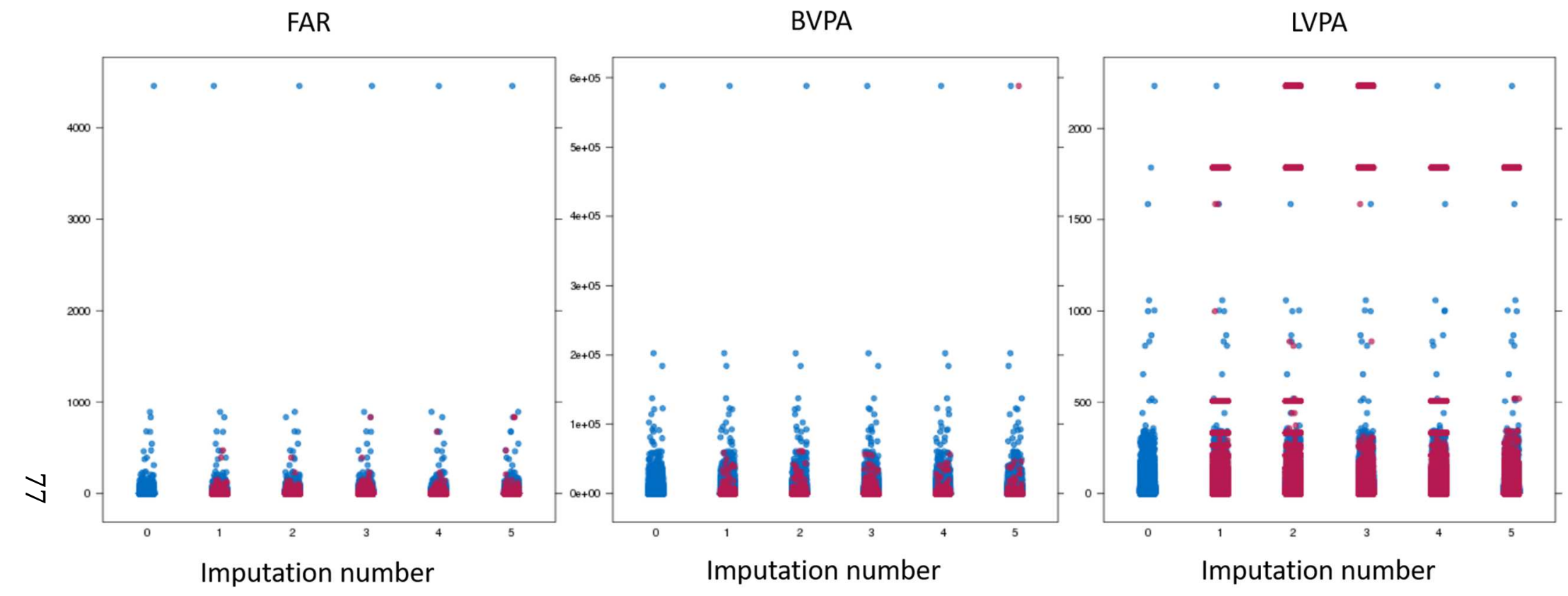

Figure 4-1 Visual Diagnosis of the CART-based MICE 


\subsection{Assessment of MICE}

To evaluate the performance of MICE, we compare the predictive accuracy of the three different MICE specifications (i.e., MICE via PMM, MICE via CART, and MICE via Random Forests). The combination of MICE with PMM is widely used because as a semi-parametric approach it can handle any variable types. Especially, PMM is often used to impute a semi-continuous missing variable. It is expected that CART and Random Forests can automatically capture complex interaction effects on missing variables with little tuning effort needed in developing the imputation models.

To this end, we first create a validation set by removing parcels with any missing values from the original parcel data, which produces a validation data set of 227,591 parcels with the 8 complete variables. Then, from the validation data set, we artificially make 5 percent of the values in the four variables (i.e., FAR, BVPA, LVPA, and LU) to be missing at random (MAR), yielding a training set of the same number of parcels but with missing values. As shown in Table 3, the artificial missing values are evenly scattered across all the missing variables, indicating an arbitrary pattern of missing data. 
Table 4-3 Missing Data Pattern of 227,591 Parcels in the Training Data Set

\begin{tabular}{|c|c|c|c|c|c|c|c|c|c|}
\hline & Area & District & POP10 & JOB11 & FAR & LVPA & $\mathrm{LU}$ & BVPA & Frequency \\
\hline & 1 & 1 & 1 & 1 & 1 & 1 & 1 & 1 & 185,350 \\
\hline & 1 & 1 & 1 & 1 & 1 & 1 & 0 & 1 & 9,745 \\
\hline & 1 & 1 & 1 & 1 & 0 & 1 & 1 & 1 & 9,711 \\
\hline & 1 & 1 & 1 & 1 & 1 & 1 & 1 & 0 & 9,883 \\
\hline & 1 & 1 & 1 & 1 & 1 & 0 & 1 & 1 & 9,729 \\
\hline & 1 & 1 & 1 & 1 & 0 & 1 & 0 & 1 & 502 \\
\hline & 1 & 1 & 1 & 1 & 1 & 1 & 0 & 0 & 532 \\
\hline & 1 & 1 & 1 & 1 & 0 & 1 & 1 & 0 & 505 \\
\hline & 1 & 1 & 1 & 1 & 1 & 0 & 0 & 1 & 512 \\
\hline & 1 & 1 & 1 & 1 & 0 & 0 & 1 & 1 & 502 \\
\hline & 1 & 1 & 1 & 1 & 1 & 0 & 1 & 0 & 516 \\
\hline & 1 & 1 & 1 & 1 & 0 & 1 & 0 & 0 & 19 \\
\hline & 1 & 1 & 1 & 1 & 0 & 0 & 0 & 1 & 33 \\
\hline & 1 & 1 & 1 & 1 & 1 & 0 & 0 & 0 & 26 \\
\hline & 1 & 1 & 1 & 1 & 0 & 0 & 1 & 0 & 26 \\
\hline Total & none & none & none & none & 11,298 & 11,344 & 11,369 & 11,507 & 227,591 \\
\hline
\end{tabular}

With this training data set, we perform MICE with three different imputation models (i.e., PMM, CART, and Random Forests). The 'mice' package of $\mathrm{R}$ is used for the PMM-based MICE and the CART-based MICE (Van Buuren \& Groothuisoudshoorn, 2011), while we use the 'CALIBERrfimpute' package of R for the MICE imputation based on Random Forests (Shah, 2014). Each of the three MICE imputation runs produces 5 imputed data sets as designed. It is often suggested that small numbers of imputed data sets (e.g., 3 or 5) are sufficient unless missing rates are unusually high, say $50 \%$ (White et al, 2010). For each imputed data set, we compare the imputed values of the training set with the original complete values of the validation set. We calculate root mean square error (RMSE) and R-squared for the continuous variables, whereas an accuracy rate is computed for the categorical variable (Fawcett, 2006). 
Table 4 shows the validation results. We find that both of the non-parametric MICE imputations perform much better than the semi-parametric MICE for the categorical missing variable (i.e., LU), indicated by a higher average accuracy rate for MICE via Random Forest (0.934) and MICE via CART (0.927) than MICE via PMM (0.780). However, it is found that there are noticeable differences in performance between the two non-parametric imputations for the continuous missing variables. The MICE with Random Forests performs best for LVPA; the average R-squared for LVPA is 0.450 with Random Forests, 0.426 with CART, and 0.234 with PMM. As for FAR and BVPA, the MICE with CART performs best with the highest average R-square $(0.665$ for FAR and 0.506 for BVPA). Surprisingly, the Random Forests within MICE shows a similar performance to the PMM within MICE (0.568 and 0.569 for FAR; 0.405 and 0.406 for BVPA). The lower performance of Random Forests in this study can be explained by a different level of skewness for the three continuous missing variables. As mentioned earlier, as the three variables are semi-continuous, they are all severely positive or right skewed. However, there is a significant gap in the skewness level among the semi-continuous missing variables. The skewness of FAR, BVPA, and LVPA in the training data set is 65,222 , and 16 , respectively. The relatively high skewness, especially of BVPA, results from some extreme data points or outliers as shown in the blue dots of Figure 1. Since we build only 10 trees in Random Forests due to computational burden, it is possible to easily miss the effects of such extreme values during the tree building process of drawing bootstrap samples and selecting random input variables. Therefore, 
we conclude that the CART-based MICE is the most appropriate for imputing missing values in a large data set, such as the parcel data in this study.

Table 4-4 Validation Results of MICE (PMM vs. CART vs. Random Forests)

\begin{tabular}{|c|c|c|c|c|c|c|c|c|}
\hline \multirow{2}{*}{$\begin{array}{c}\text { Imputation } \\
\text { Model }\end{array}$} & \multirow{2}{*}{$\begin{array}{l}\text { Missing } \\
\text { Variable }\end{array}$} & \multirow{2}{*}{$\begin{array}{l}\text { Validation } \\
\text { Measure }\end{array}$} & \multicolumn{5}{|c|}{ Multiple Imputed Data Set } & \multirow{2}{*}{ Average } \\
\hline & & & $m=1$ & $\mathrm{~m}=2$ & $m=3$ & $m=4$ & $m=5$ & \\
\hline \multirow{7}{*}{ PMM } & \multirow{2}{*}{ FAR } & RMSE & 0.270 & 0.286 & 0.268 & 0.294 & 0.283 & 0.280 \\
\hline & & R-squred & 0.590 & 0.558 & 0.586 & 0.547 & 0.565 & 0.569 \\
\hline & \multirow{2}{*}{ BVPA } & RMSE & 50.871 & 38.446 & 51.229 & 41.432 & 34.979 & 43.391 \\
\hline & & R-squred & 0.285 & 0.457 & 0.181 & 0.453 & 0.656 & 0.406 \\
\hline & \multirow{2}{*}{ LVPA } & RMSE & 19.157 & 17.104 & 17.184 & 17.657 & 16.662 & 17.553 \\
\hline & & R-squred & 0.179 & 0.250 & 0.236 & 0.232 & 0.276 & 0.234 \\
\hline & LU & $\begin{array}{c}\text { Accuracy } \\
\text { Rate }\end{array}$ & 0.780 & 0.783 & 0.781 & 0.776 & 0.782 & 0.780 \\
\hline \multirow{7}{*}{ CART } & \multirow{2}{*}{ FAR } & RMSE & 0.247 & 0.222 & 0.248 & 0.238 & 0.238 & 0.239 \\
\hline & & R-squred & 0.684 & 0.710 & 0.637 & 0.655 & 0.641 & 0.665 \\
\hline & \multirow{2}{*}{ BVPA } & RMSE & 43.843 & 34.327 & 30.682 & 34.442 & 45.009 & 37.661 \\
\hline & & R-squred & 0.331 & 0.571 & 0.670 & 0.563 & 0.393 & 0.506 \\
\hline & \multirow{2}{*}{ LVPA } & RMSE & 14.484 & 15.269 & 14.306 & 13.801 & 13.955 & 14.363 \\
\hline & & R-squred & 0.422 & 0.384 & 0.427 & 0.454 & 0.443 & 0.426 \\
\hline & LU & $\begin{array}{c}\text { Accuracy } \\
\text { Rate } \\
\end{array}$ & 0.927 & 0.925 & 0.930 & 0.924 & 0.928 & 0.927 \\
\hline \multirow{7}{*}{$\begin{array}{c}\text { Random } \\
\text { Forest }\end{array}$} & \multirow{2}{*}{ FAR } & RMSE & 0.284 & 0.313 & 0.282 & 0.281 & 0.229 & 0.278 \\
\hline & & R-squred & 0.543 & 0.506 & 0.559 & 0.553 & 0.676 & 0.568 \\
\hline & \multirow{2}{*}{ BVPA } & RMSE & 76.119 & 73.102 & 36.429 & 36.961 & 33.766 & 51.275 \\
\hline & & R-squred & 0.195 & 0.187 & 0.541 & 0.515 & 0.585 & 0.405 \\
\hline & \multirow{2}{*}{ LVPA } & RMSE & 14.110 & 13.435 & 13.692 & 13.953 & 14.978 & 14.034 \\
\hline & & R-squred & 0.446 & 0.479 & 0.467 & 0.450 & 0.409 & 0.450 \\
\hline & LU & $\begin{array}{c}\text { Accuracy } \\
\text { Rate }\end{array}$ & 0.936 & 0.934 & 0.933 & 0.935 & 0.932 & 0.934 \\
\hline
\end{tabular}

\subsection{Discussion}

In this paper, we have demonstrated MICE as a flexible method to implement multiple imputation for the multivariate missing values in parcel data. We consider MICE via PMM, and two recursive portioning techniques (i.e., CART and Random Forests) as imputation engines for MICE. To assess their performance, we have conducted crossvalidation and found that, under limited computing power, the CART-based MICE has 
performed best for imputing missing values in both continuous and discrete variables, especially when the distribution of the continuous variables to be imputed is skewed. For future research, we plan to improve the performance of Random Forestsbased MICE by increasing the number of trees, which is expected to produce more reliable imputers. In addition, although we have generated multiple imputed data sets, we have not taken full advantage of them. We plan to conduct standard statistical analyses with the resulting multiple imputed data sets and assess the uncertainty introduced via imputed data. Lastly, this study focuses primarily on recursive partitioning methods as an imputation model. There are many other machine learning techniques, such as k-nearest neighbor, support vector machine, and Bayesian network that have been used in data imputation. It would be interesting to benchmark them with the methods in this study. 


\section{CONCLUSION}

Travel forecasting models have been developed to support informed decision-making about alternative transportation and land use scenarios. The key benefit of activity-based models is that they allow the assessment of a wider range of planning and policy than trip-based models. Especially, activity-based models can provide significantly more robust insights into policies and projects that may affect individuals' activity and travel behavior as they are implemented in a fully disaggregate microsimulation framework. Some examples include congestion pricing, parking pricing, flexible work schedules, a variety of transit fare policies, and so on.

However, activity-based travel forecasting is still in a formative stage. Existing U.S. activity-based models are not fully capable of evaluating such modern transportation and land use policies. One possible solution for having more policy-sensitivity would be to maximize the level of details in terms of market segmentation, temporal scale and spatial resolution, which is one of the most critical design considerations for activitybased modeling (Castiglione et al., 2015). Unlike trip-based models, adding more demographic segments, more time periods, and more zones does not substantially influence run time and data storage in activity-based models. This dissertation explores recent methodological advances to take full advantage of this key feature of activitybased models.

First, activity-based models simulate individual households and persons in a synthetic population. A variety of socio-demographic characteristics of residents in the 
modeling area are used to be representative of the actual population. With all the combinations of standard variables, thousands of different types of agents can be considered in activity-based models, rather than just a couple of demographic segments in trip-based models. On the other hand, individuals' daily activity pattern is so complex due to its multidimensionality that researchers need a method to examine the daily patterns from a holistic point of view. Recently sequence alignment was introduced in the field of travel behavior research to answer such an "old" question. In Chapter 2, an innovative method was proposed by linking sequence alignment with ANODI (Analysis of Distance). It was shown that this new methodological combination can identify unique attributes of travelers that influence complex daily activity-travel patterns. Likewise, the proposed method can be used to detect policy-sensitive variables for simulated individuals. In addition, since activity-based models provide more detailed outputs with a list of individual households, persons, tours and trips, their application for environmental justice analysis becomes more common to understand the impacts of transportation scenarios on different population groups (Cheng, Hu, Huang, \& Wen, 2012). The proposed method can be applied for environment justice analysis in a different way by examining daily activity and travel patterns as a whole rather than specific accessibility and mobility indicators.

Second, activity-based models explicitly represent time-of-day choices, such as activity arrival and departure times as well as activity duration. Continuous-time duration models are typically used for activity duration analysis. Chapter 3 discussed some limitations of the continuous-time framework and proposed a discrete-time duration 
model that is essentially a simplest form of dynamic discrete choice models. One of the key benefits is that the discrete-time approach can easily handle time-varying constraints and allow to track household members and cars for each of 10 or 15 -min discrete-time periods. Similarly, the most recent version of CT-RAMP explicitly traces car use at origin and destination of each trip (Vovsha, et al., 2017). The temporal detail is critical given that the integration of activity-based models with dynamic traffic assignment becomes increasingly important. A strong potential of the proposed method is that the activity-based demand model can be dynamic or time-dependent as well, so that the integration with the dynamic supply model can result in "truly emergent" travel forecasting.

Third, it has become more common to use multiple spatial scales in activity-based models. For example, traffic analysis zones may be large enough for auto and transit skimming, while microzones or parcels may reduce aggregation bias to measure accessibilities associated with population, employment, school enrollment, parking supply, distance to transit stops, urban density, etc. Using a smaller spatial scale is increasingly important because transportation and land use projects pay more attention to their local-level impacts. However, it is challenging to develop and maintain small-scale spatial information, especially for future-year scenarios. In Chapter 3, multiple imputation by chained equations was introduced to handle multivariate missing values in parcel data. This sophisticate data imputation technique was adopted to develop a continuous and reusable data hub for land use and transportation planning and modeling (Wang \& Kim, 2014). 
As the age of Big Data has come, travel forecasting has a tremendous opportunity to move forward to its mature status. Big Data, such as cellular tower data, navigationGPS data, and Location Base-Services (LBS) data, allows us to observe and understand mobility behavior at the level of details that has never happened before (Anda, Fourie, \& Erath, 2016). Nevertheless almost all existing activity-based travel demand models rely on conventional data sources, such as household travel surveys and census data. The three innovative methods proposed in this dissertation may help realize the unprecedented level of details by allowing for greater flexibility in the model specification. 


\section{REFERENCES}

Abayomi, K., Gelman, A., \& Levy, M. (2008). Diagnostics for Multivariate Imputations. Journal of the Royal Statistical Society: Series C (Applied Statistics), 57(3), 273297.

Abbott, A., \& Tsay, A. (2000). Sequence Analysis and Optimal Matching Methods in Sociology. Sociological Methods \& Research, 29(3), 3-33.

Aisenbrey, S., \& Fasang, A. (2010). New Life for Old Ideas: The 'Second Wave' of Sequence Analysis Bringing the 'Course' Back Into the Life Course. Sociological Methods \& Research, 38(3), 420-462.

Anda, C., Fourie, P., \& Erath, A. (2016). Transport Modelling in the Age of Big Data. Singapore-ETH Centre.

Anderson, M. (2001). A New Method for Non-Parametric Multivariate Analysis of Variance. Austral Ecology, 26(1), 32-46.

Azur, M., Stuart, E., Frangakis, C., \& Leaf, P. (2011). Multiple Imputation by Chained

Equations: What is it and How does it Work? International Journal of Methods in Psychiatric Research, 20(1), 40-49.

Bhat, C. (1996). A Hazard-Based Duration Model of Shopping Activity with Nonparametric Baseline Specification and Nonparametric Control for Unobserved Heterogeneity. Transportation Research Part B, 30(3), 189-207.

Bhat, C., \& Pinjari, A. R. (2008). Duration Modeling. In D. Hensher, \& K. Button (Eds.), Handbook of Transport Modeling. Elsevier.

Bonetti, M., Piccarreta, R., \& Salford, G. (2013). Parametric and Nonparametric Analysis of Life Courses: An Application to Family Formation Patterns. Demography, 50(3), 881-902.

Bradley, M., Bowman, J., \& Lawton, K. (1999). A Comparison of Sample Enumeration and Stochastic Microsimulation for Application of Tour-Based and ActivityBased Travel Demand Models. European Transport Conference. Cambridge, UK.

Breiman, L. (2001). Random Forests. Machine Learning, 45, 5-32.

Breiman, L., Friedman, J., Olshen, R., \& Stone, C. (1984). Classification and Regression Trees. Monterey, CA, USA: Wadsworth \& Brooks.

Burgette, L. F., \& Reiter, J. P. (2010). Multiple Imputation for Missing Data via Sequential Regression Trees. American Journal of Epidemiology, 172(9), 10701076.

Burnett, P., \& Hanson, S. (1982). The Analysis of Travel as an Example of Complex Human Behavior in Spatially Constrained Situations: Definition and Measurement Issues. Transportation Research Part A, 16(2), 87-102.

Castiglione, J., Bradley, M., \& Gliebe, J. (2015). Activity-Based Travel Demand Models: A Primer. Washington D.C.: Transportation Research Board.

Cheng, H., Hu, H.-H., Huang, G., \& Wen, F. (2012). Application of Activity Based Model on Environmental Justice Analysis - a Case Study of SCAG ABM. 14th TRB National Transportation Planning Applications Conference. Columbus, Ohio. 
Davidson, W., Donnelly, R., Vovsha, P., Freedman, J., Ruegg, S., Hicks, J., . . Picado, R. (2007). Synthesis of First Practices and Operational Research Approaches in Activity-Based Travel Demand Modeling. Transportation Research Part A, 41(5), 464-488.

Doherty, S., \& Mohammadian, A. (2011). The Validity of Using Activity Type to Structure Tour-Based Scheduling Models. Transportation, 38(1), 45-63.

Donders, A., van der Heijden, G., Stijnen, T., \& Moons, K. (2006). Review: a Gentle Introduction to Imputation of Missing Values. Journal of Clinical Epidemiology, 59(10), 1087-1091.

Donnelly, R., Erhardt, G., Moeckel, R., \& Davidson, W. (2010). NCHRP Synthesis 406 Advanced Practices in Travel Forecasting. Washington D.C.: Tranportation Research Board.

Doove, L., Van Buuren, S., \& Dusseldrop, E. (2014). Recursive Partitioning for Missing Data Imputation in the Presence of Interaction Effects. Computational Statistics and Data Analysis, 72, 92-104.

Eskelson, B., Temesgen, H., Lemay, V., Barrett, T., Crookston, N., \& Hudak, A. (2009). The Roles of Nearest Neighbor Methods in Imputing Missing Data in Forest Inventory and Monitoring Databases. Scandinavian Journal of Forest Research, 24(3), 235-246.

Ettema, D. (1996). Activity-Based Travel Demand Modeling. PhD Dissertation. Netherlands: Technische Universiteit Eindhoven.

Ettema, D., Borgers, A., \& Timmermans, H. (1995). Competing Risk Hazard Model of Activity Choice, Timing, Sequencing, and Duration. Transportation Research Record: Journal of the Transportation Research Board(1493), 101-109.

Fawcett, T. (2006). An Introduction to ROC Analysis. Pattern Recognition Letters, 27(8), 861-874.

Gabadinho, A., Ritschard, G., Muller, N., \& Studer, M. (2011). Analyzing and Visualizing State Sequences in R with TraMineR. Journal of Statistical Software, 40(4), 1-37.

Gliebe, J., \& Kim, K. (2010). Time-Dependent Utility in Activity and Travel Choice Behavior. Transportation Research Record: Journal of the Transportation Research Board(2156), 9-16.

Heckman, J., \& Navarro, S. (2007). Dynamic Discrete Choice and Dynamic Treatment Effects. Journal of Econometrics, 136(2), 341-396.

Joh, C.-H., Arentze, T., \& Timmermans, H. (2001). Pattern Recognition in Complex Activity Travel Patterns: Comparison of Euclidean Distance, Signal-Processing Theoretical, and Multidimensional Sequence Alignment Methods. Transportation Research Record: Journal of the Transportation Research Board(1752), 16-22.

Joh, C.-H., Arentze, T., Hofman, F., \& Timmermans, H. (2002). Activity Pattern Similarity: A Multidimensional Sequence Alignment Method. Transportation Research Part B, 36(5), 385-403.

Kim, K. (2014). Discrepancy Analysis of Activity Sequences: What Does Explain the Complexity of People's Daily Activity-Travel Patterns? Transportation Research Record: Journal of the Transportation Research Board(2413), 24-33. 
Konduri, K. C. (2012). Integrated Model of the Urban Continuum with Dynamic Timedependent Activity-Travel Microsimulation: Framework, Prototype, and Implementation.

Koppelman, F., \& Pas, E. (1985). Travel-Activity Behavior in Time and Space: Methods for Representation and Analysis. In P. Nijkamp, H. Leitner, \& N. Wrigley (Eds.), Measuring the Unmeasurable (pp. 587-627). Dordrecht, The Netherlands: Martinus Nijhoff.

Lakshminarayan, K., Harp, S., \& Samad, T. (1999). Imputation of Missing Data in Industrial Databases. Applied Intelligence, 11(3), 259-275.

Lesnard, L. (2010). Setting Cost in Optimal Matching to Uncover Contemporaneous Socio-Temporal Patterns. Sociological Methods \& Research, 38(3), 389-419.

Martin, P., \& Wiggins, R. (2011). Optimal Matching Analysis. In M. Williams, \& P. Vogt, The SAGE Handbook of Innovation in Social Research Methods (pp. 385408). SAGE Publications.

McArdle, B., \& Anderson, M. (2001). Anderson. Fitting Multivariate Models to Community Data: A Comment on Distance-Based Redundancy Analysis. Ecology, 82(1), 290-297.

Paleti, R., Vovsha, P., Picado, R., Alexandr, B., Hu, H.-H., \& Huang, G. (2015). Development of Time Varying Accessibility Measures: Application to the Activity-Based Model for Southern California. the 94th Annual Meeting of TRB. Washington D.C.: Transportation Research Board.

Pas, E. (1983). A Flexible and Integrated Methodology for Analytical Classification of Daily Travel-Activity Behavior. Transportation Science, 17(4), 405-429.

Pas, E. (1997). Recent Advances in Activity-Based Travel Demand Modeling. ActivityBased Travel Forecasting Conference (pp. 79-102). Arlington: Texas Transportation Institute.

Pinjari, A., \& Bhat, C. (2011). Activity-Based Travel Demand Analysis. In A. de Palma, R. Lindsey, E. Quinet, \& R. Vickeman, A Handbook of Transport Economics (pp. 213-248). Edward Elgar Pub.

Recker, W., McNally, M., \& Root, G. (1985). Travel/Activity Analysis: Pattern Recognition, Classification, and Interpretation. Transportation Research Part A, 19(4), 279-296.

Rubin, D. (1987). Multiple Imputation for Nonresponse in Surveys. New York: John Wiley \& Sons.

Sammour, G., Bellemans, T., Vanhoof, K., Janssens, D., Kochan, B., \& Wets, G. (2012). The Usefulness of the Sequence Alignment Methods in Validating Rule-Based Activity-Based Forecasting Models. Transportation, 39(4), 773-789.

Saneinejad, S., \& Roorda, M. (2009). Application of Sequence Alignment Methods in Clustering and Analysis of Routine Weekly Activity Schedules. Transportation Letters: The International Journal of Transportation Research, 1(3), 197-211.

Schafer, J. (1997). Analysis of Incomplete Multivariate Data. London, United Kingdon: Chapman \& Hall Ltd.

Schafer, J., \& Graham, J. (2002). Missing Data: Our View of the State of the Art. Psychological Methods, 7(2), 147-177. 
Schlich, R., \& Axhausen, K. (2003). Habitual Travel Behaviour: Evidence from a SixWeek Travel Diary. Transportation, 20(1), 13-36.

Shah, A. (2014). CALIBERrfimpute: Imputation in MICE using Random Forest. Retrieved from http://cran.rproject.org/web/packages/CALIBERrfimpute/index.html

Shah, A., Bartlett, J., Carpenter, J., Nicholas, O., \& Hemingway, H. (2014). Comparison of Random Forest and Parametric Imputation Models for Imputing Missing Data Using MICE: a CALIBER Study. American Journal of Epidemiology, 179(6), 764-774.

Shoval, N., \& Isaacson, M. (2007). Sequence Alignment as a Method for Human Activity Analysis in Space and Time. Annals of the Association of American Geographers, 97(2), 282-297.

Singer, J., \& Willett, J. (2003). Applied Longitudinal Data Analysis: Modeling Change and Event Occurrence. New York: Oxford University Press.

Steele, F. (2005). Event History Analysis. Bristol: ESRC National Centre for Research Methods.

Steele, F. (2008). Multilevel Models for Longitudinal Data. Journal of the Royal Statistical Society: Series A (Statistics in Society), 4(2), 5-19.

Steele, F. (2011). Multilevel Discrete-Time Event History Models with Applications to the Analysis of Recurrent Employment Transitions. Australian and New Zealand Journal of Statistics, 53(1), 1-26.

Steele, F., Diamond, I., \& Wang, D. (1996). The Determinants of the Duration of Contraceptive Use in China: A Multi-level Multinomial Discrete-Hazards Modeling Approach. Demography, 33(1), 12-23.

Steele, F., Goldstein, H., \& Browne, W. (2004). A General Multilevel Multistate Competing Risks Model for Event History Data, with an Application to a Study of Contraceptive Use Dynamics. Statistical Modelling, 4(2), 145-159.

Studer, M., Ritschard, G., Gabadinho, A., \& Müller, N. (2011). Discrepancy Analysis of State Sequences. Sociological Methods \& Research, 40(3), 471-510.

Van Buuren, S. (2007). Multiple Imputation of Discrete and Continuous Data by Fully Conditional Specification. Statistical Methods in Medical Research, 16(3), 219242.

Van Buuren, S., \& Groothuis-oudshoorn, K. (2011). mice : Multivariate Imputation by Chained Equations in R. Journal of Statistical Software, 45(3).

Vovsha, P., \& Bradley, M. (2004). A Hybrid Discrete Choice Departure Time and Duration Model for Scheduling Tours. Transportation Research Record: Journal of the Transportation Research Board(1894), 46-56.

Vovsha, P., Bradley, M., \& Bowman, J. (2005). Activity-Based Travel Forecasting Models in the United States: Progress since 1995 and Prospects for the Future. In H. Timmermans (Ed.), Progress in Activity-Based Analysis (pp. 389-414). Amsterdam, the Netherlands: Elsevier.

Vovsha, P., Hicks, J., Vyas, G., Livshits, V., Jeon, K., Anderson, R., \& Giaimo, G. (2017). Combinatorial Tour Mode Choice. 96th Annual Meeting of the Transportation Research Board. Washington D.C. 
Waddell, P. (2009). Parcel-Level Microsimulation of Land Use and Transportation: The Walking Scale of Urban Sustainability. Resource Paper for the 2009 IATBR Workshop.

Waddell, P., Peak, C., \& Caballero, P. (2004). UrbanSim: Database Development for the Puget Sound Region. Seattle: Center for Urban Simulation and Policy Analysis, University of Washington.

Wang, L., \& Kim, K. (2014). Continuous Data Integration for Land Use and Transportation Planning and Modeling. National Institue for Transportation and Communities.

Wegener, M. (2011). From Macro to Micro - How Much Micro is too Much? Transport Reveiws, 31(2), 161-177.

White, I., Royston, P., \& Wood, A. (2010). Multiple Imputation Using Chained Equations: Issues and Guidance for Practice. Statistics in Medicine, 30(4), 377399.

Wilson, C. (1998). Analysis of Travel Behavior Using Sequence Alignment Methods. Transportation Research Record: Journal of the Transportation Research Board(1645), 52-59.

Wilson, C. (2001). Activity Patterns of Canadian Women: Application of ClustalG Sequence Alignment Software. Transportation Research Record: the Journal of the Transportation Research board, 1777, 55-67.

Wilson, C. (2008). Activity Patterns in Space and Time: Calculating Representative Hagerstrand Trajectories. Transportation, 35(4), 485-499. 